\title{
Magnoliófitas presentes en el humedal de Castilla, Piura - Perú
}

\section{RESUMEN}

\author{
Vicky Almendra Correa Seminario \\ vcorrea@unf.edu.pe \\ https://orcid.org/0000-0003-2642-9385
}

Universidad Nacional de Frontera

Jesús Manuel Charcape Ravelo jcharcaper@unp.edu.pe

Scopus Author ID: 57204357611 https://orcid.org/0000-0002-9595-1663 Universidad Nacional de Piura

Claudia Mabel Palacios Zapata cmpalacios@unf.edu.pe https://orcid.org/0000-0001-5738-5384

Universidad Nacional de Frontera

William Lorenzo Aldana Juárez waldana@unf.edu.pe https://orcid.org/0000-0003-4079-0601

Universidad Nacional de Frontera

Los humedales son cruciales para la vida por las funciones que desarrollan y por los servicios ecosistémicos que nos proveen, pero muchos están colapsando por la acción antrópica. El objetivo de la investigación fue conocer la composición florística de este ecosistema. El humedal se ubica en el distrito de Castilla, Piura, tiene una superficie de 34.65 hectáreas. Se realizaron ocho exploraciones botánicas cada dos meses durante el 2019 al 2020, se fotografiaron las especies in situ; se llevaron en la prensa de madera al laboratorio de Botánica para su determinación. Se indican, el nombre científico, autor, año de publicación, familia, nombre vulgar y el hábito. Se reportan 50 especies de Magnoliófitas, contenidas en 20 familias, Asteraceae es la familia más numerosa con 8 especies; seguida de Fabaceae y Poaceae con 6 especies, y las demás familias con 5 a menos. La especie dominante en las orillas fangosas fue Bacopa monnieri y en el terreno fueron Colicodendron scabridum y Tamarix aphylla. El número elevado de especies presentes en el humedal nos indican que es un ecosistema muy importante en flora a pesar de encontrarse en medio del Desierto Superárido Premontano Tropical.

Palabras clave: desierto costero, fanerógamas, flora, angiopermas, Piura. 


\title{
Magnoliophytes present in the wetland of Castilla, Piura - Peru
}

\begin{abstract}
Wetlands are crucial to life for the functions they perform and for the ecosystem services they provide us, but many are collapsing due to human action. The objective of the research was to know the floristic composition of this ecosystem. The wetland is located in the Castilla district, Piura, it has an area of 34.65 hectares. Eight botanical explorations were carried out every two months during 2019 to 2020, the species were photographed in situ; they were taken in the wood press to the Botany laboratory for determination. The scientific name, author, year of publication, family, common name and habit are indicated. 50 species of Magnoliophytes are reported, contained in 20 families, Asteraceae is the largest family with 8 species; followed by Fabaceae and Poaceae with 6 species, and the other families with 5 less. The dominant species on the muddy shores was Bacopa monnieri and on the ground they were Colicodendron scabridum and Tamarix aphylla. The high number of species present in the wetland indicates that it is a very important ecosystem in flora despite being in the middle of the Superarid desertPremontane Tropical.
\end{abstract}

Keywords: coastal desert, phanerogams, flora, angiosperms, Piura.

Artículo recibido: 15 febrero 2021 Aceptado para publicación: 10 marzo 2021

Correspondencia: vcorrea@unf.edu.pe Conflictos de Interés: Ninguna que declarar 


\section{INTRODUCCIÓN}

Los humedales se definen por la presencia de agua, ya sea de manera permanente o estacional, y adquieren características de ecosistemas distintos; sobresaliendo por su gran productividad y diversidad, equiparándose con las selvas tropicales (Kusler et al., 1994). Los humedales son zonas de transición entre ecosistemas de tierras altas y acuáticas, otros se forman en depresiones de las montañas que acumulan agua o en zonas bajas donde el agua subterránea aflora a la superficie. En ellos, la presencia del agua es determinante en toda la biogeoquímica de un área, esto es, en las características biológicas, físicas y químicas de un sitio en particular. El factor principal que distingue a los humedales de otras formas terrestres o cuerpos de agua es la vegetación particular que se adapta a las condiciones únicas de su suelo; ya que, éstos ecosistemas consisten principalmente en suelo hídrico, que sustenta a las plantas acuáticas y terrestres de su zona de influencia (Charcape \& Moutarde, 2005; Mitsch \& Gosselink, 2007 y Butler, 2010).

Podemos describir fácilmente las cualidades e importancia de los humedales, se debate mucho sobre cómo definirlos correctamente. Una definición adecuada se volvió crucial para clasificar a éstos con fines legales, y poder determinar qué tierras están protegidas por la legislación estatal y federal; por ello, el Servicio de Pesca y Vida Silvestre de USA en 1979 definió los definió así: “Los humedales son tierras de transición entre sistemas terrestres y acuáticos donde el nivel freático suele estar en la superficie o cerca de ella, o la tierra está cubierta por aguas poco profundas... Los humedales deben tener uno o más de los siguientes tres atributos: 1) al menos periódicamente, la tierra soporta predominantemente hidrofitos; 2) el sustrato es predominantemente suelo hídrico no drenado; y 3) el sustrato está saturado de agua o cubierto por agua poco profunda en algún momento durante la temporada de crecimiento de cada año" (Cowardin, et al., 1979). Pero, la definición más usada, es la del convenio Ramsar, en el artículo 1.1 del protocolo, dice que son humedales: "las extensiones de marismas, pantanos y turberas, o superficies cubiertas de aguas, sean éstas de régimen natural o artificial, permanentes o temporales, estancadas o corrientes, dulces, salobres o saladas, incluidas las extensiones de agua marina cuya profundidad en marea baja no exceda de seis metros" (Ramsar, 1971).

En el mundo, los datos exactos sobre el área que ocupan los humedales están aumentando. En el mundo, los humedales continentales y costeros cubren más de 12,1 millones de $\mathrm{km}^{2}$, un área mayor que la de Canadá, con un $54 \%$ inundado de forma permanente y un $46 \%$ 
de manera estacional. Sin embargo, los humedales naturales están disminuyendo a largo plazo en todo el mundo: entre 1970 y 2015, tanto los humedales continentales como los marinos y costeros disminuyeron en aproximadamente un 35\%, en los casos en los que se disponía de datos, una tasa tres veces superior a la de pérdida de bosques. Por el contrario, los humedales artificiales, en su mayoría arrozales y embalses, casi se duplicaron durante este período y ahora constituyen el $12 \%$ de los humedales. Estos aumentos no han compensado la pérdida de humedales naturales (Ramsar, 2018).

Los ecosistemas de humedales son fundamentales para el ciclo de vida de plantas y animales, son hábitat de muchas especies de flora y fauna, sirven de refugio y suministro temporal de las aves migratorias. Los de gran tamaño tienen una función global moderando los cambios climáticos, actuando de sumideros de $\mathrm{CO}_{2}$, como las turberas de Canadá, Alaska y Eurasia. Amenguan el golpe de las olas y almacenan las aguas de inundación; retienen el sedimento y reducen la contaminación, por ello los llaman los "riñones del planeta". Producen alimentos, sirven para cultivos lucrativos como el arroz silvestre, cazamos animales peces y mariscos; también son fuente de forraje para los animales domésticos y silvestres (Kusler et al. 1994).

Las especies dependientes de los humedales, como las plantas, peces, aves acuáticas y tortugas, experimentan una grave disminución, una cuarta parte de ellas están amenazadas de extinción, especialmente en los trópicos. Desde 1970, el $81 \%$ de las poblaciones de especies de humedales continentales y el $36 \%$ de las especies costeras y marinas han disminuido. Los niveles de amenaza mundial son altos (>10\%) para casi todos los taxones dependientes de humedales continentales y costeros evaluados. Los niveles más altos de amenaza de extinción (> 30\%) de varias especies de fauna. El riesgo de extinción va en aumento. Los humedales sanos y naturales son fundamentales para la supervivencia humana; sin embargo, éstos enfrentan un gran número de desafíos (Ramsar, 2018).

En Perú se distinguen cinco tipos de humedales: Marinos (humedales costeros, lagunas costeras, costas rocosas y arrecifes de corales), estuarinos (deltas, marismas de marea y manglares), lacustres (humedales asociados con lagos), ribereños (humedales adyacentes a ríos y arroyos) y, palustres (pantanos, marismas y ciénagas). Las principales amenazas son: crecimiento urbano, avance de la agricultura sin ordenamiento, contaminación, aprovechamiento ilegal y el cambio climático global; pero el mayor y principal enemigo a enfrentar es la presión por cambio de uso que drena sus aguas (SERFOR, 2015). 
Los humedales de la costa peruana están a lo largo del litoral marítimo, siendo de aguas marinas, dulces y salobres dependiendo de la ubicación de los humedales, son continentales (interiores), marinos (colindantes al litoral), salobres (colindantes al litoral con presencia de aguas dulces-ríos, marinas-mar). Asimismo, se categorizan en naturales y artificiales (construidos por el hombre). Estos ambientes son de gran importancia por la belleza paisajística que poseen, la concentración de una alta diversidad biológica, y por la por la posibilidad de utilización de recursos naturales, aunque muchos de ellos han desaparecido (ProNaturaleza, 2010).

La división Magnoliophyta, es la más amplia del reino vegetal, formada por plantas con flores, que son unas 369000 spp. de hierbas, arbustos, árboles y otras formas, adaptadas a casi cualquier hábitat. Tenemos dos clases: Magnoliopsida (o Dicotiledóneas), y Liliopsida (o Monocotiledóneas) que son los grupos más extensos y variados de plantas con flores llamado también Angiospermas (RBG, 2016).

La gran mayoría de trabajos en humedales continentales costeros de Piura, están enfocados al estudio de la fauna, en especial de las aves, faltando información sobre las especies vegetales. A su vez, un inventario florístico es la herramienta empleada por los botánicos para conocer la flora y vegetación de un territorio; esta diversidad florística es catalogada es un parámetro correcto que cuantifica el valor natural de un ecosistema; estos conocimientos precisos de la flora servirán para delimitar los objetivos de uso, gestión y conservación de los valores naturales del humedal. Por este motivo, el objetivo de esta investigación fue determinar las especies de magnoliófitas o angiospermas presentes en el humedal de Castilla - Piura, ecosistema que no está protegido y ha sido muy poco estudiado.

\section{MATERIALES Y MÉTODOS}

El humedal de Castilla se encuentra ubicado entre los 5¹4'22" - 80³6'27"O; al Sureste de la ciudad de Piura, en el distrito de Castilla; y comprende la Zona de vida: desierto superárido - Premontano Tropical (ds-PT) de Perú, esta zona se caracteriza por tener un clima desértico, la temperatura media anual fluctúa entre $\operatorname{los} 23^{\circ} \mathrm{C}$ a $23.6^{\circ} \mathrm{C}$, las precipitaciones pluviales anuales son de 30 a $50 \mathrm{~mm}$. $(<250 \mathrm{~mm})$. El promedio de la Relación de Evapotranspiración Potencial total anual varía de 16 a 32; es decir, tiene un gran déficit de humedad en el suelo (Tosi, 1960). Este humedal tiene una superficie de 34.65 ha, que parecerían ser casi dos cuerpos de agua por una reducción o estrechamiento 
en su zona central, es una pequeña laguna formada en una depresión del desierto, rodeada por terrenos de cultivo de empresas agro exportadoras y poblados rústicos, sometido por lo tanto a un fuerte impacto antrópico.

Figura 01: Ubicación del humedal de Castilla - Piura.

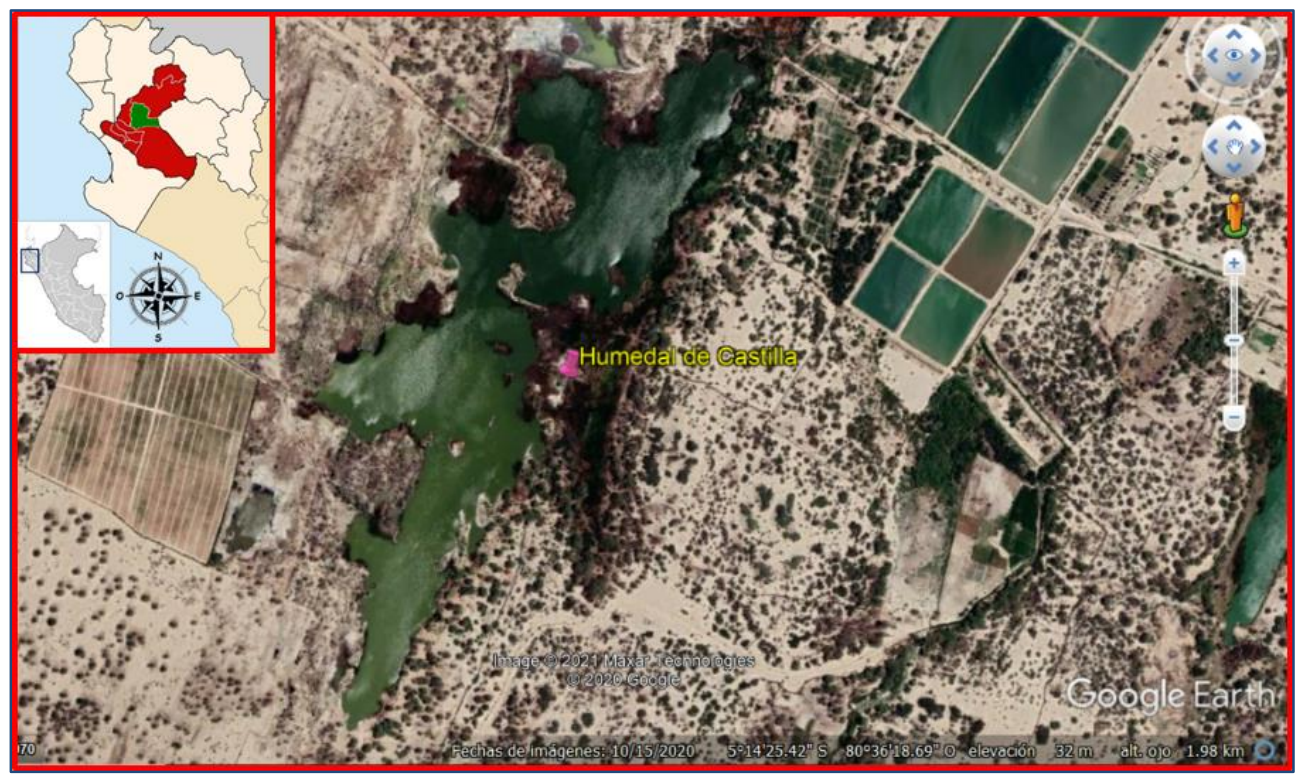

En el recuadro pequeño de la izquierda, en gris se muestran a Perú y la región Piura, de rojo a la provincia de Piura y de verde al distrito de Castilla donde se encuentra el humedal. Tomado de una imagen satelital de Google Earth, 2020.

Para el estudio de la flora, se realizaron ocho exploraciones botánicas, cada dos meses a este humedal, desde mayo de 2019 a julio de 2020; en el lugar, se empleó el método ad libitum, que significa a placer o como guste, es decir se trató de recorrer toda el área haciendo una búsqueda intensiva de las especies (spp.) para encontrar la mayor cantidad de plantas posibles. A los especímenes se los fotografiaron in situ, se registró el hábito, hábitat de las plantas y se realizaron colectas, utilizando tijeras podadoras y las técnicas estándar de colecta botánica (Bridson \& Forman, 1992). La mayoría de especies se determinaron en el lugar, otras fueron llevadas en la prensa de madera al laboratorio de Botánica Fanerogámica de la Universidad Nacional de Piura para su determinación definitiva, empleando claves botánicas y bibliografía especializada (León, 1993; León, Cano \& Young, 1995 y Mostacero et al., 2009), además de consultas a especialistas del Herbarium Piurense. La clasificación y nomenclatura botánica se basó en The Angiosperm Phylogeny Group IV (APG IV, 2016). 


\section{RESULTADOS Y DISCUSIÓN}

Se reportan un total de 50 especies, las cuales están contenidas en 42 géneros y 20 familias. A continuación, se muestra la lista alfabética de las especies de magnoliófitas reportadas en el humedal de Castilla, Piura - Perú, se indican el nombre científico, el autor, año de publicación, familia botánica y sus nombres comunes o vulgares.

1) Acacia nilotica (L.) Willd. ex Delile 1813 Fabaceae "faique" (Fig. 31)

2) Ageratum conyzoides (L.) L. 1753 Asteraceae "huarmi huarmi" (Fig. 20)

3) Alternanthera halimifolia (Lam.) Pitt. 1926 Amaranthaceae "hierba blanca" (Fig. 05)

4) Amaranthus celosioides Kunth 1817 Amaranthaceae "yuyo" (Fig. 06)

5) Amaranthus hybridus L. 1753 Amaranthaceae "yuyo hembra" (Fig. 07)

6) Amaranthus spinosus L. 1753 Amaranthaceae "yuyo macho" (Fig. 08)

7) Bacopa monnieri (L.) Wettstein 1891 Plantaginaceae "bacopa" (Fig. 39)

8) Bidens pilosa L. 1753 Asteraceae "amor seco" (Fig. 21)

9) Beautempsia avicenniifolia (Kunth) Gaudich. 1866 Capparaceae "sapote” (Fig. 17)

10) Capparicordis crotonoides (Kun.) Iltis \& Cor. 2007 Capparaceae "vichayo" (Fig. 18)

11) Cenchrus echinatus L. 1753 Poaceae "cadillo" (Fig. 45)

12) Cenchrus myosuroides Kunth 1815 Poaceae "cadillo", "falso cadillo" (Fig. 46)

13) Chloris virgata Swartz 1797 Poaceae "gramilla"

14) Colicodendron scabridum (Kunth) Seemann 1852 Capparaceae "sapote" (Fig. 19)

15) Conyza bonariensis (L.) Cronquist 1943 Asteraceae "yuyo moro" (Fig. 22)

16) Cryptocarpus pyriformis Kunth 1817 Nyctaginaceae "chope" (Fig. 30)

17) Cynanchum formosum Nicholas Brown 1895 Apocynaceae "bejuco" (Fig. 11)

18) Cynodon dactylon (L.) Persoon 1805 Poaceae "grama dulce" (Fig. 47)

19) Cyperus rotundus L. 1753 Cyperaceae "coquito"

20) Dactyloctenium aegyptium (L.) Willdenow 1809 Poaceae "estrella” (Fig. 48)

21) Desmanthus virgatus (L.) Willdenow 1806 Fabaceae "espadita de Pizarro" (Fig. 33)

22) Exodeconus maritimus (Bentham) D'Arcy 1986 Solanaceae "campanilla" (Fig. 43)

23) Grabowskia boerhaaviifolia (L.f.) Schlech. 1832 Solanaceae "palo negro" (Fig. 38)

24) Heliotropium angiospermum Murr. 1770 Boraginaceae "hierba del alacrán” (Fig. 13)

25) Heliotropium curassavicum L. 1753 Boraginaceae "hierba del alacrán” (Fig. 14)

26) Macroptilium atropurpureum (DC.) Urban 1928 Fabaceae "citrato" (Fig. 34)

27) Momordica charantia L. 1753 Cucurbitaceae "papayilla" (Fig. 28) 
28) Parkinsonia aculeata L. 1753 Fabaceae "nuche", "azote de Cristo" (Fig. 35)

29) Portulaca oleracea Linnaeus 1753 Portulacaceae "verdolaga" (Fig. 40)

30) Proboscidea altheifolia (Bent.) Dec. 1865 Martyniaceae "yuca de caballo" (Fig. 29)

31) Prosopis pallida (H. \& B. ex Willd.) Kunth 1823 Fabaceae "algarrobo" (Fig. 36)

32) Sarcocornia fruticosa (L.) A. J. Scott 1978 Amaranthaceae "parachique" (Fig. 09)

33) Scutia spicata (H. \& B. ex Willd.) Weberbauer 1930 Rhamnaceae "peal"

34) Solanum americanum Miller 1768 Solanaceae "hierba mora"

35) Solanum pimpinellifolium L. 1755 Solanaceae "tomatillo" (Fig. 37)

36) Sonchus asper (L.) Hill 1769 Asteraceae “cerraja”, "cerrajón” (Fig. 23)

37) Sonchus oleraceus L. 1753 Asteraceae “cerraja” (Fig. 24)

38) Spilanthes leiocarpa DC. 1836 Asteraceae "turre macho" (Fig. 25)

39) Spilanthes urens Jacquin 1760 Asteraceae "turre macho", "turre” (Fig. 26)

40) Tamarix aphylla (L.) H. Karsten 1882 Tamaricaceae “casuarina”, "támariz” (Fig. 41)

41) Tessaria integrifolia Ruiz \& Pavón 1798 Asteraceae "pájaro bobo" (Fig. 27)

42) Tiquilia dichotoma (R. \& P.) Pers. 1805 Boraginaceae "flor de arena" (Fig. 15)

43) Tiquilia paronychioides (Phil.) Rich. 1976 Boraginaceae "manito de ratón” (Fig. 16)

44) Trianthema portulacastrum L. 1753 Aizoaceae "verdolaga de hoja ancha" (Fig. 10)

45) Tribulus terrestris L. 1753 Zygophyllaceae “abrojo” (Fig. 42)

46) Typha angustifolia L. 1753 Typhaceae "totora", “enea” (Fig. 44)

47) Vachellia macracantha (H. \& B. ex W.) Se. \& Eb. 2005 Fabaceae "faique" (Fig. 32)

48) Vallesia glabra (Cavanilles) Link 1821 Apocynaceae "cun cun” (Fig. 12)

49) Washingtonia filifera (Lin. ex A.) Wend. ex Bary 1880 Arecaceae "palmera"

50) Zea mays L. 1753 Poaceae "maíz"

Figura 02: Composición de las Magnoliófitas del Humedal de Castilla.

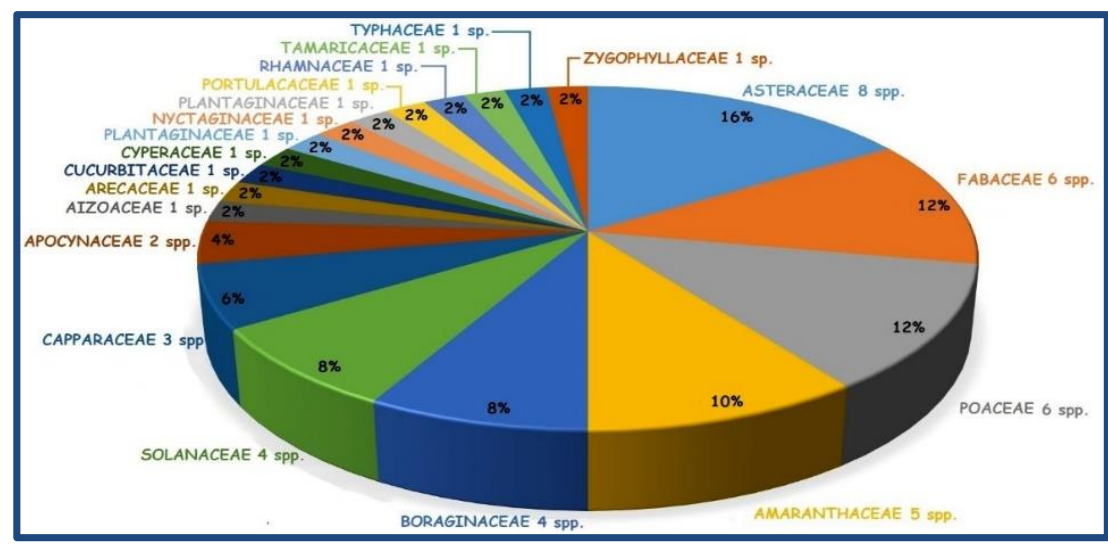


Asteraceae es la familia más numerosa con 8 spp., que representan el 16\%. Le siguen Fabaceae y Poaceae ambas con 6 spp., 12\% cada una; las Amaranthaceae con 5 spp. (10\%); Boraginaceae y Solanaceae ambas con 4 spp. (8\% c/u); Capparaceae con 3 spp. (6\%); Apocynaceae con $2 \mathrm{spp}$. (4\%); les siguen las siguientes 12 familias todas con 1 sola especie ( $2 \% \mathrm{c} / \mathrm{u}$ ) (Figura 2). Nuestros resultados concuerdan en gran parte con el trabajo de Monzón \& Peláez (2015) para el humedal costero de "Tres Palos”, quienes reportaron 51 especies en 21 familias de plantas vasculares, siendo las Asteraceae y Poaceae las familias más numerosas con 8 spp. c/u, seguida de las Fabaceae con 6 spp. y las Amaranthaceae con 3 spp. Para el caso de las Fabaceae, según SOTWP, 2017, esta familia contiene $24505 \mathrm{spp}$. siendo la tercera familia con mayor riqueza de especies después de las Asteraceae (32 913 spp.) y Orchidaceae (27 801 spp.), esta familia es además una de las más representativas de estas zonas desérticas.

Sin embargo, esta investigación, difiere con las investigaciones de León et al., (1995) en los "Pantanos de Villa", donde reportan 55 spp.; ACRAMM (2016) para la "Albufera de Medio Mundo", donde reportan 26 spp., e INRENA (2004) en la “Alburera Paraíso" que dan a conocer 33 spp., sus familias con mayor número de spp. fueron Poaceae y Cyperaceae, pero con un menor número de spp. a pesar de tener una mayor área; esto a causa de la acción antrópica de estos ecosistemas muy frágiles, donde han aprovechado y extraído las plantas de las otras familias por la utilidad que presentan; y porque el "humedal de Tres Palos" y el "Humedal de Castilla" se ubican en el norte de Perú cerca de la línea ecuatorial, mientras los otros tres humedales se encuentran ubicados en la región Lima al centro de la costa del país.

Figura 03: Presencia de especies nativas frente a las introducidas.

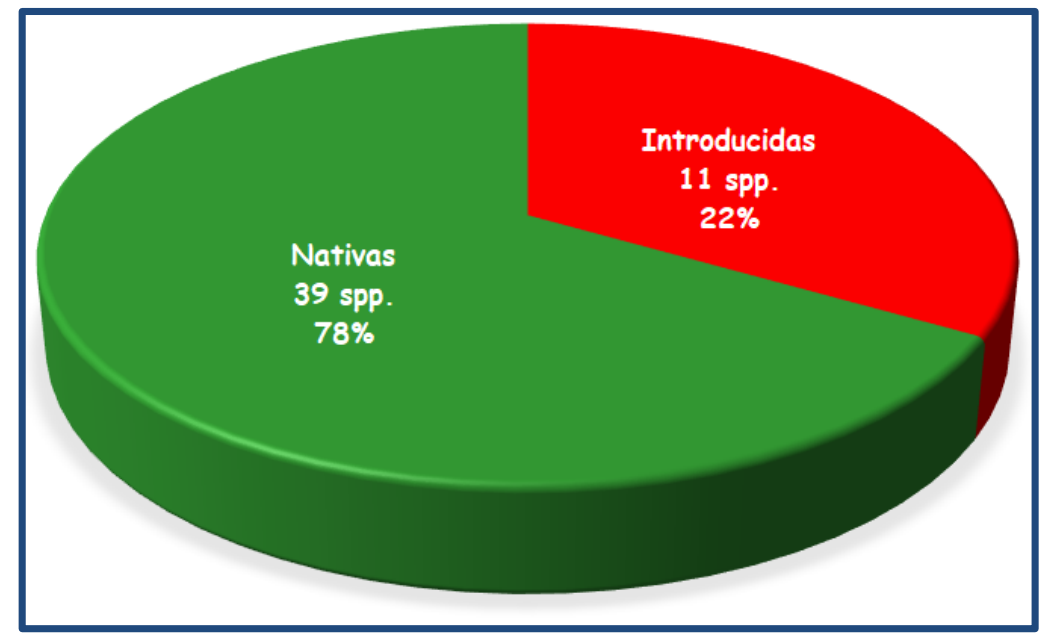


Las especies nativas aún están en mucho mayor número que las introducidas o foráneas (Figura 3); esto nos indica la manifestación de la acción antrópica; ya que cerca al humedal hay terrenos de cultivo desde donde migran las plantas foráneas para competir con las especies nativas, tal es el caso de: Portulaca oleracea, Tamarix aphylla, Tribulus terrestris, Cynodon dactylon o Dactyloctenium aegyptium, varias consideradas especies exóticas invasoras. Estas especies invasoras suelen ser uno entre un conjunto de factores que afectan a lugares o ecosistemas específicos, no siempre resulta fácil determinar la proporción que se les puede atribuir en ese impacto, como lo señala el CDB (2020).

Figura 04: Hábito de las especies de Magnoliófitas en el Humedal de Castilla.

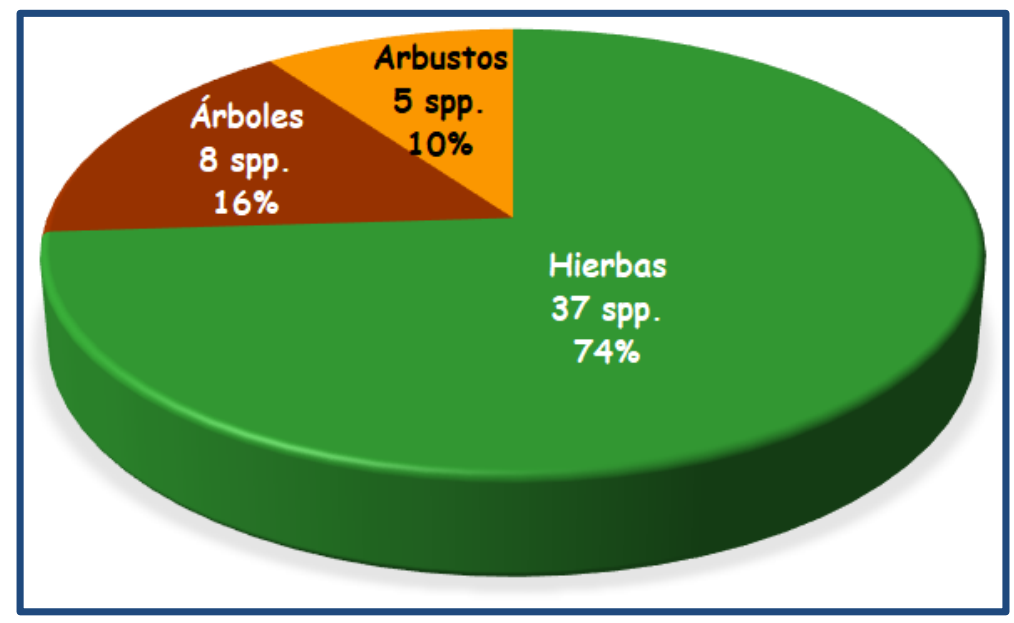

El hábito, porte o forma de vida dominante en el Humedal de Castilla, corresponde a las hierbas con 37 spp. (74\%), le siguen los árboles con 8 spp. (16\%) y finalmente los arbustos con 5 spp. (10\%) (figura 4).

Se muestran a continuación las fotografías de las especies indicando su nombre científico y familia botánica a la cual pertenecen.

Figura 05: Alternanthera halimifolia AMARANTHACEAE

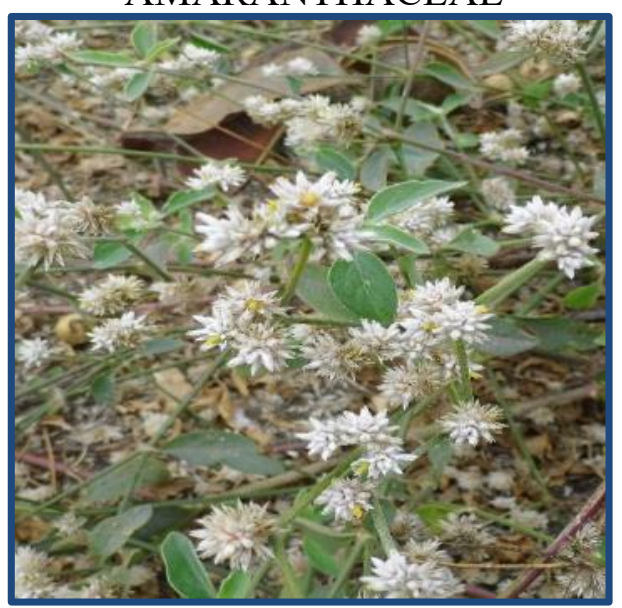

Figura 06: Amaranthus celosioides AMARANTHACEAE

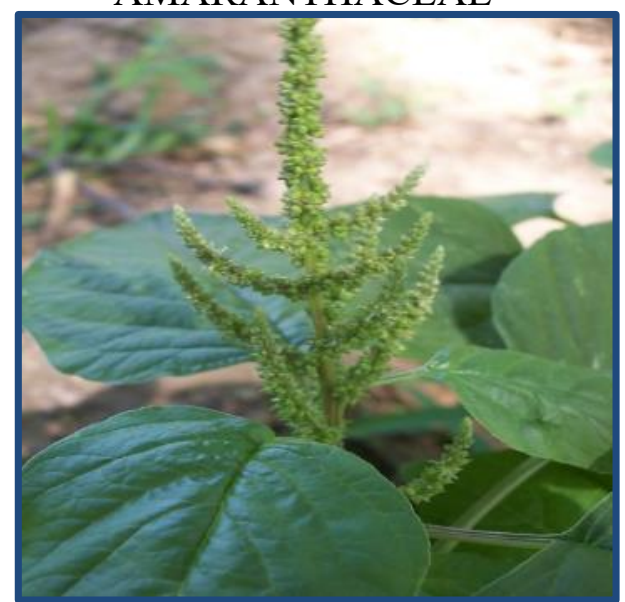

Ciencia Latina Revista Científica Multidisciplinar, Ciudad de México, México. ISSN 2707-2207 / ISSN 2707-2215 (en línea), marzo-abril, 2021, Volumen 5, Número 2. https://doi.org/10.37811/cl rcm.v5i2.337 p. 1431 
Figura 07: Amaranthus hybridus AMARANTHACEAE

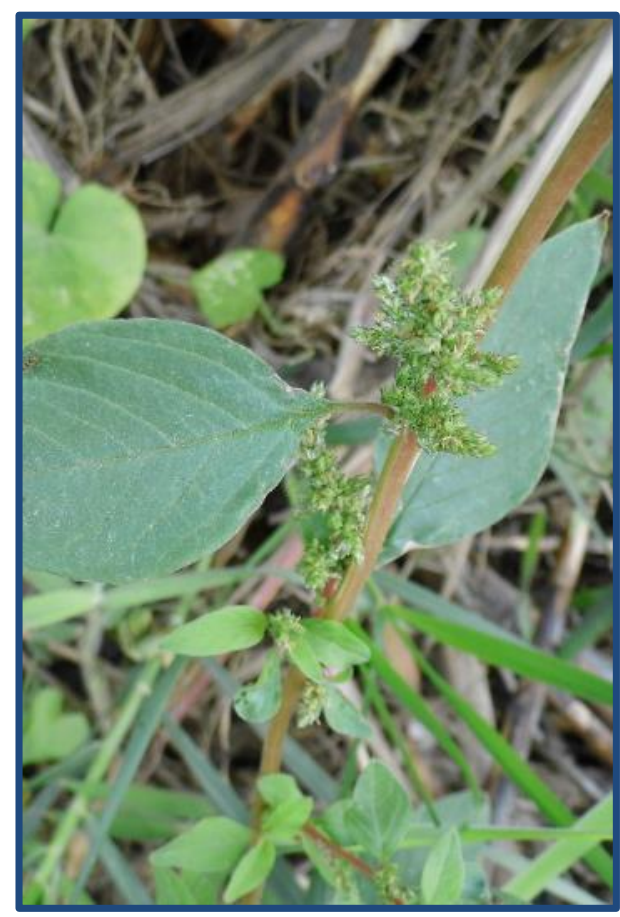

Figura 09: Sarcocornia fruticosa AMARANTHACEAE

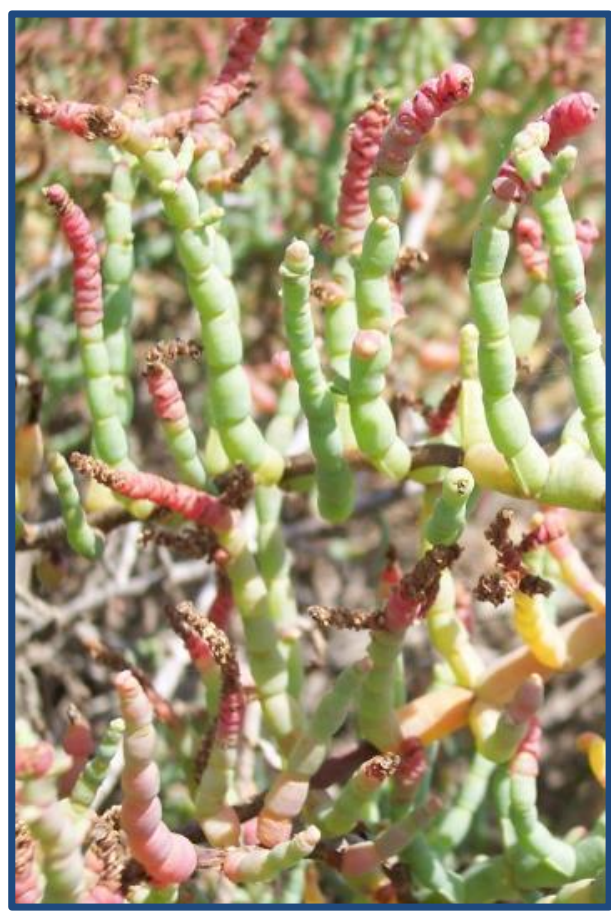

Figura 08: Amaranthus spinosus AMARANTHACEAE

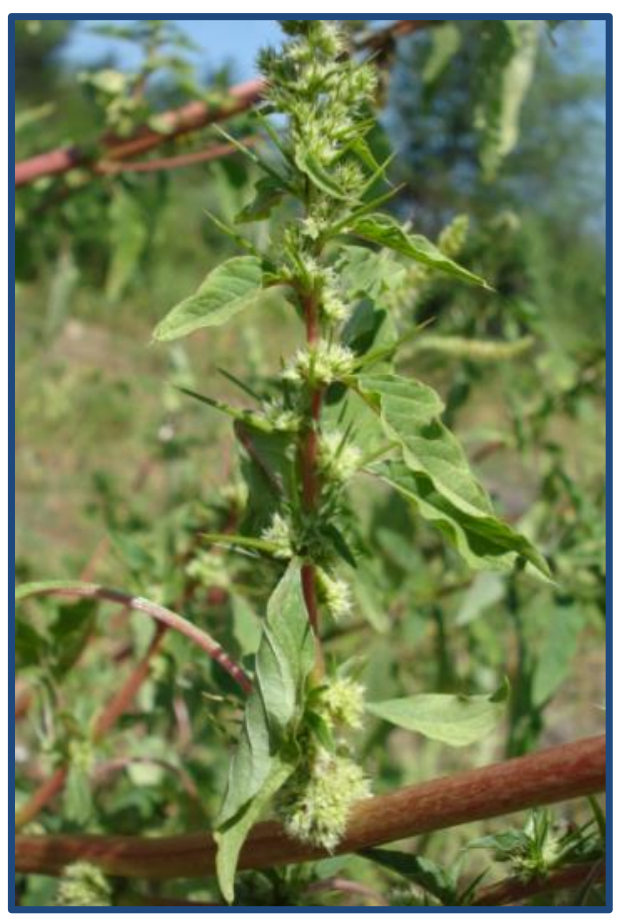

Figura 10: Trianthema portulacastrum AIZOACEAE

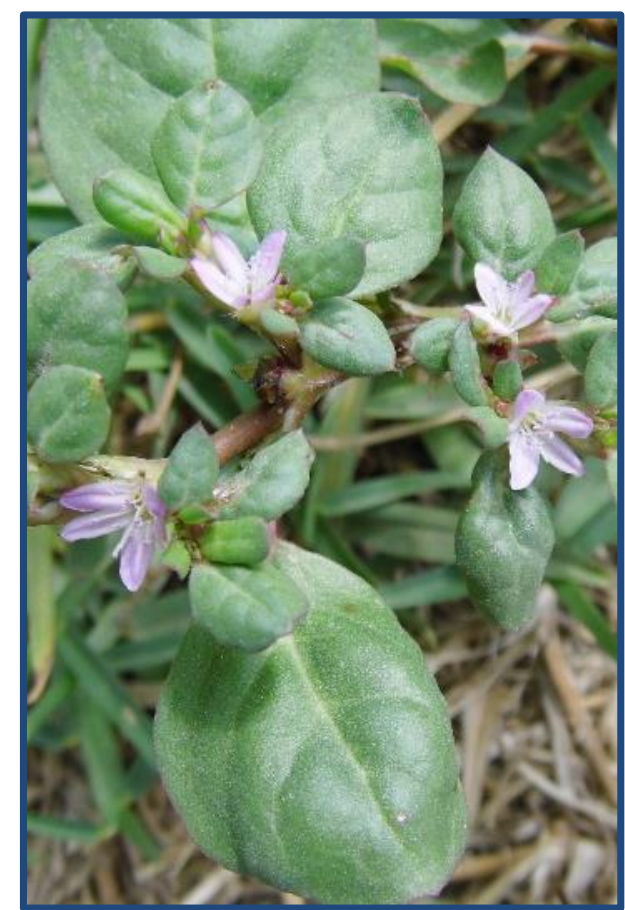


Figura 11: Cynanchum formosum APOCYNACEAE

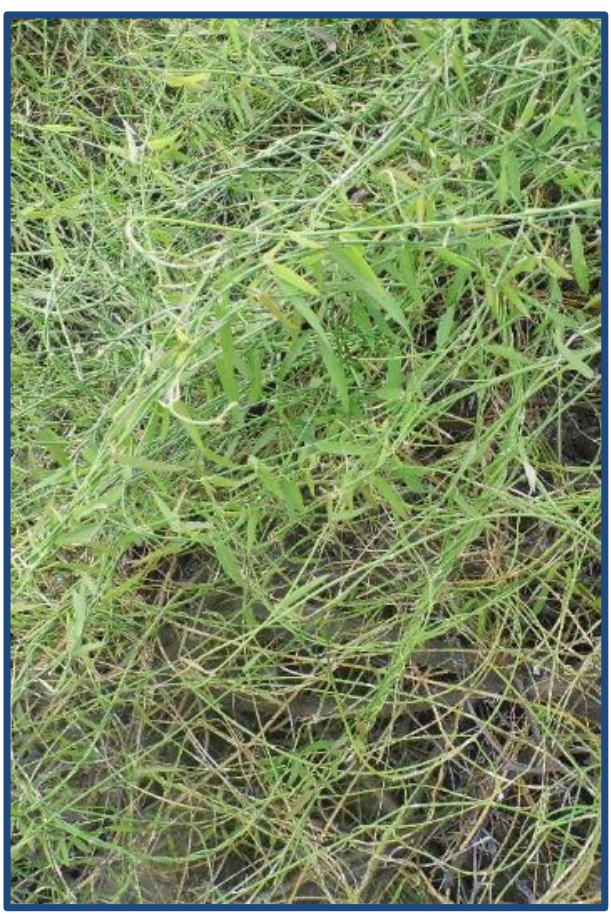

Figura 13: Heliotropium angiospermum BORAGINACEAE



Figura 12: Vallesia glabra APOCYNACEAE

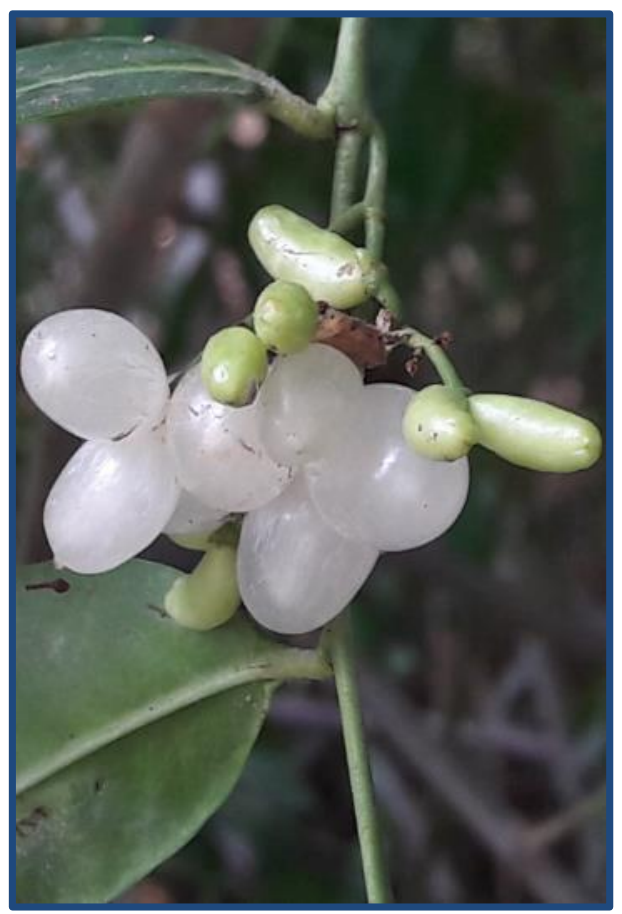

Figura 14: Heliotropium curassavicum BORAGINACEAE

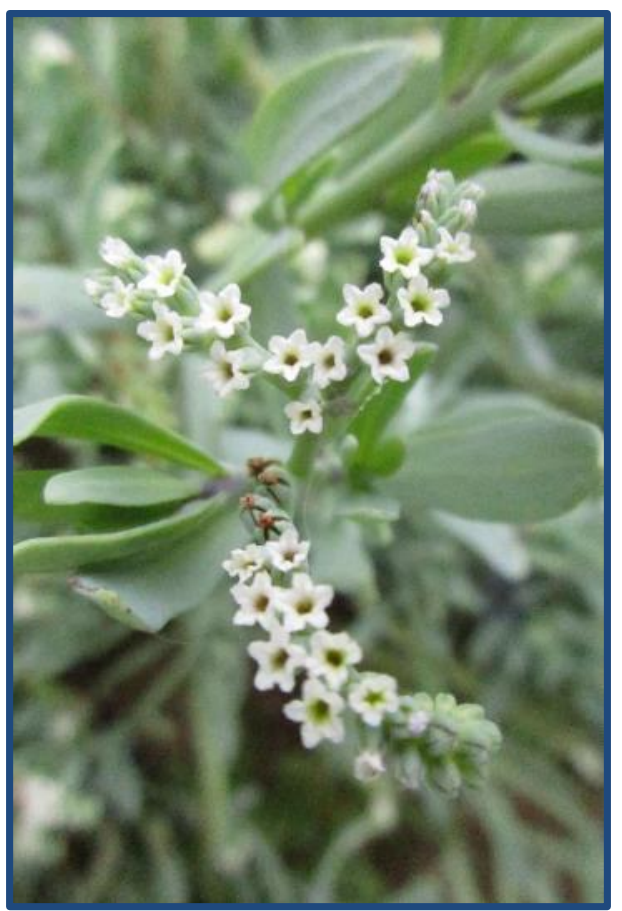

Ciencia Latina Revista Científica Multidisciplinar, Ciudad de México, México. ISSN 2707-2207 / ISSN 2707-2215 (en línea), marzo-abril, 2021, Volumen 5, Número 2. https://doi.org/10.37811/cl rem.v5i2.337 p. 1433 
Figura 15: Tiquilia dichotoma BORAGINACEAE

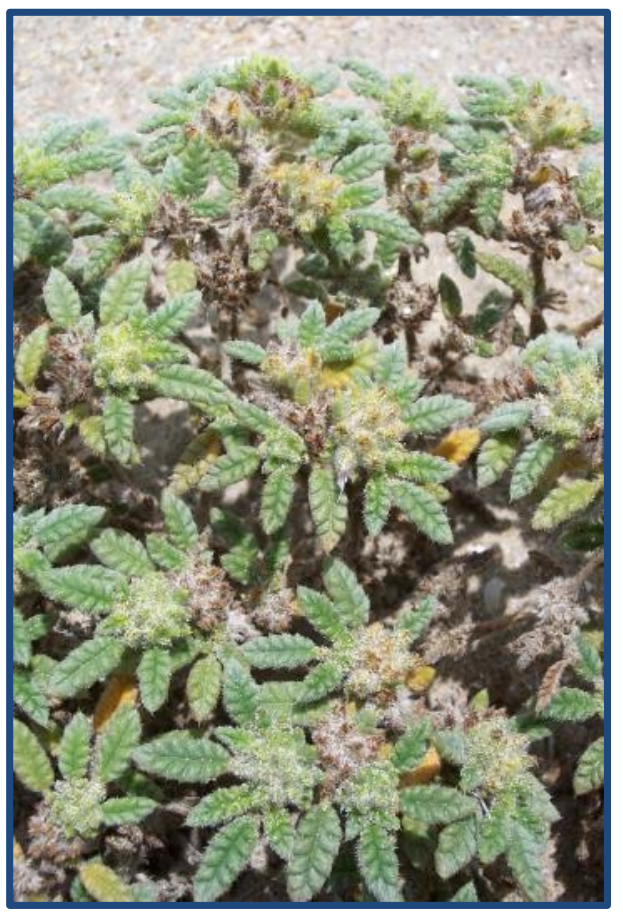

Figura 17: Beautempsia avicenniifolia CAPPARACEAE

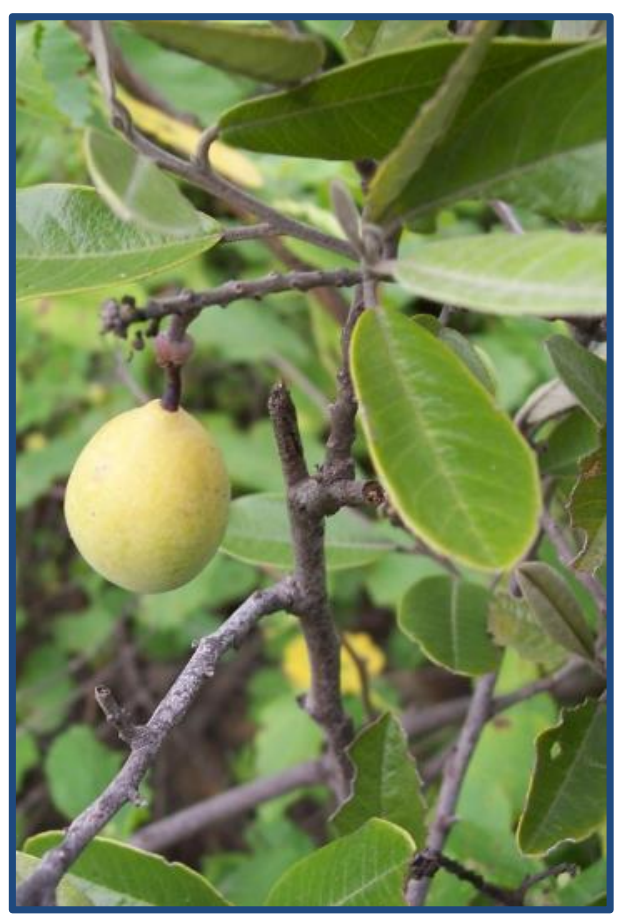

Figura 16: Tiquilia paronychioides BORAGINACEAE

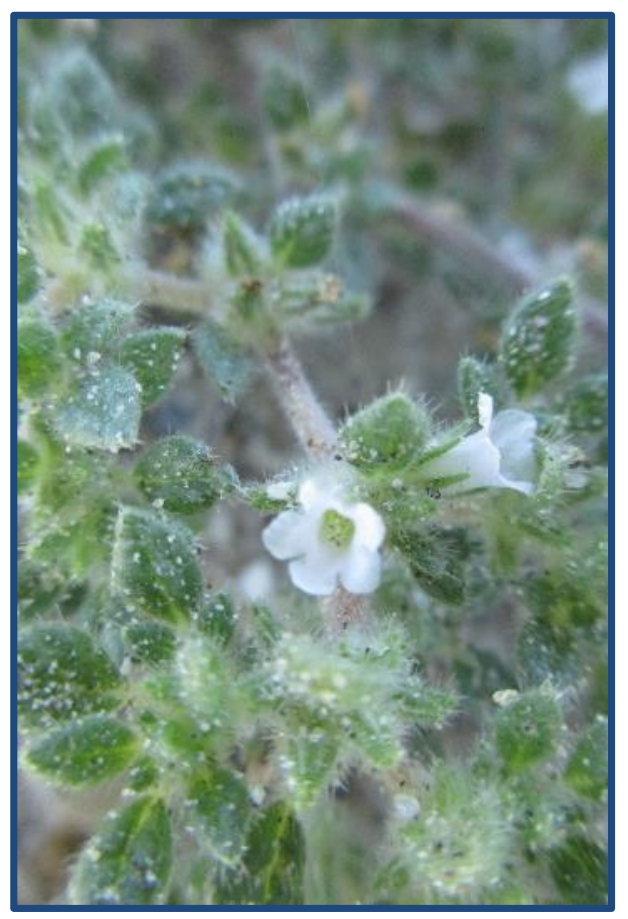

Figura 18: Capparicordis crotonoides CAPPARACEAE

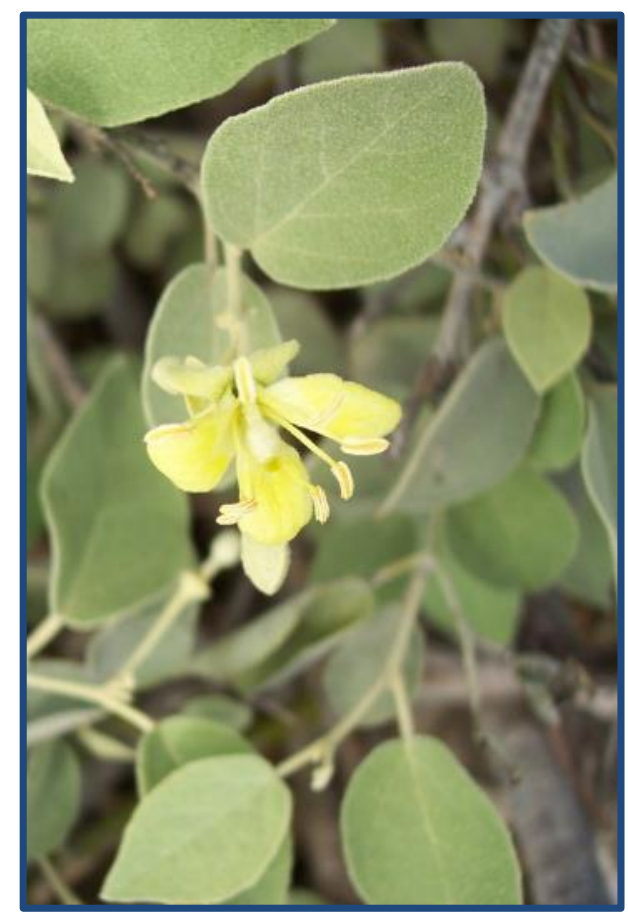

Ciencia Latina Revista Científica Multidisciplinar, Ciudad de México, México. ISSN 2707-2207 / ISSN 2707-2215 (en línea), marzo-abril, 2021, Volumen 5, Número 2. https://doi.org/10.37811/cl rcm.v5i2.337 p. 1434 
Figura 19: Colicodendron scabridum CAPPARACEAE

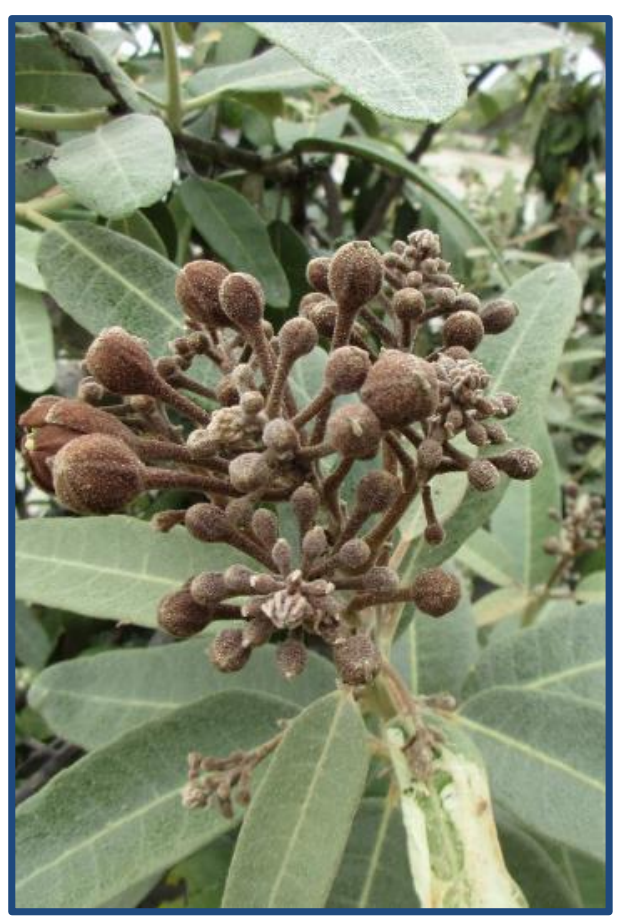

Figura 21: Bidens pilosa ASTERACEAE

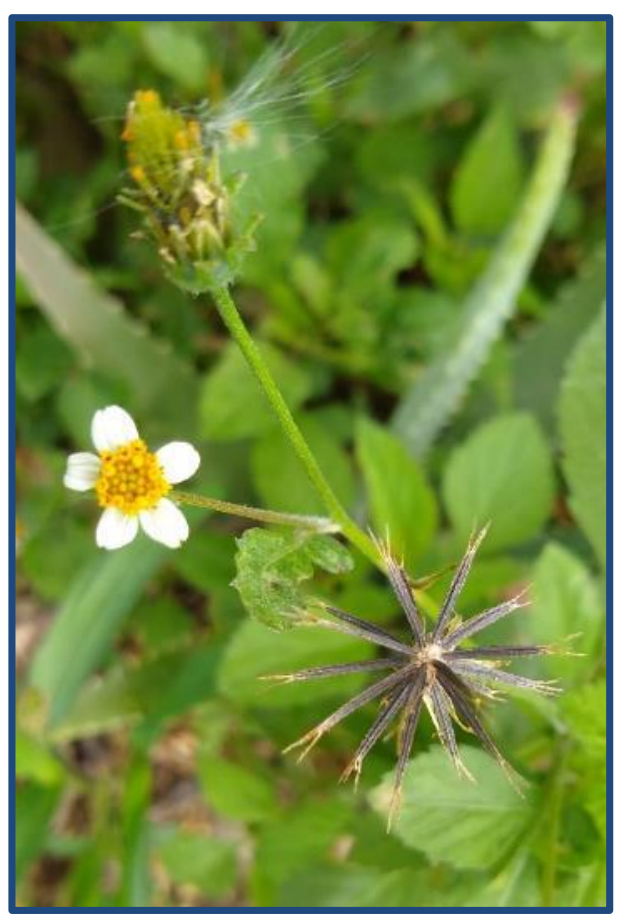

Figura 20: Ageratum conyzoides ASTERACEAE

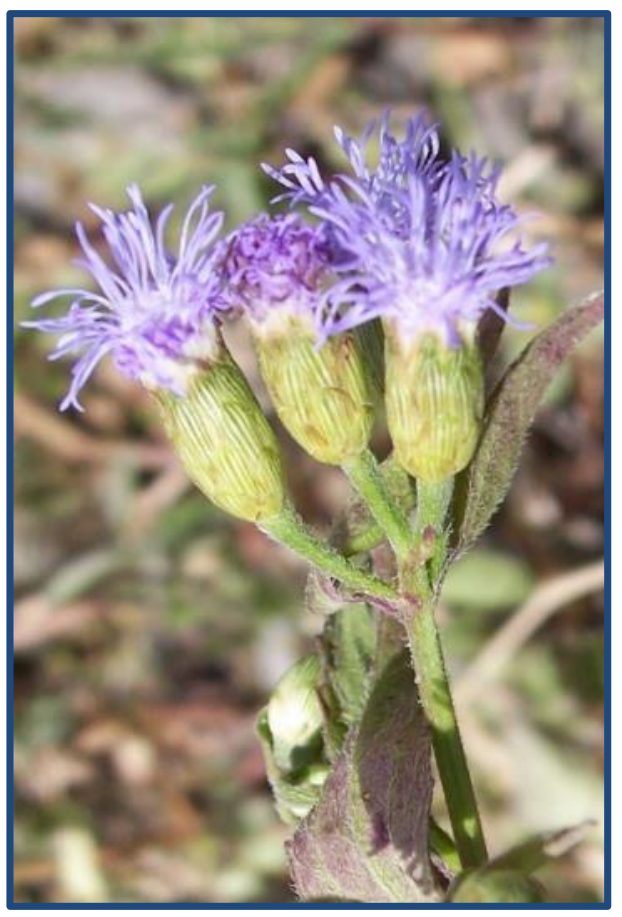

Figura 22: Conyza bonariensis ASTERACEAE

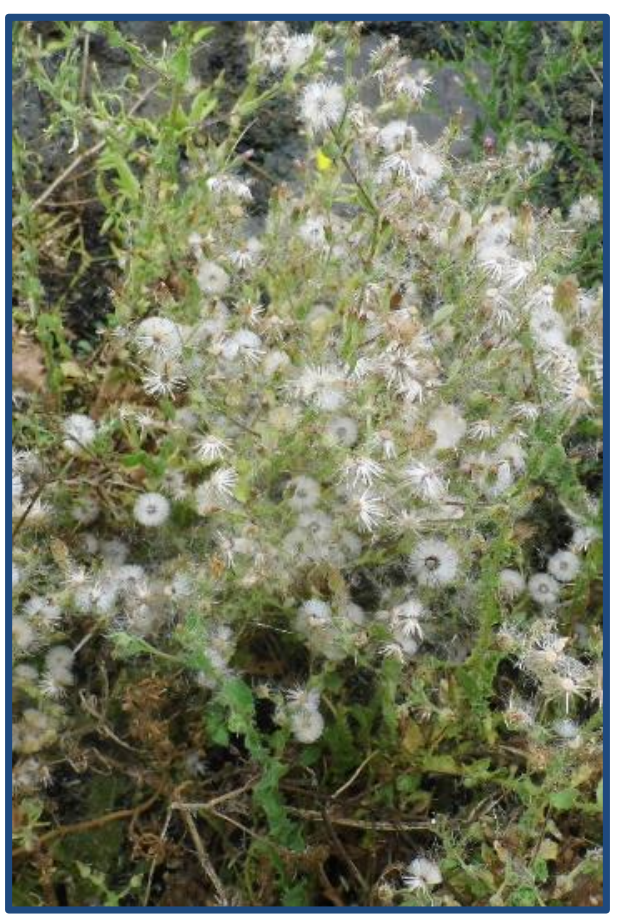


Figura 23: Sonchus asper ASTERACEAE

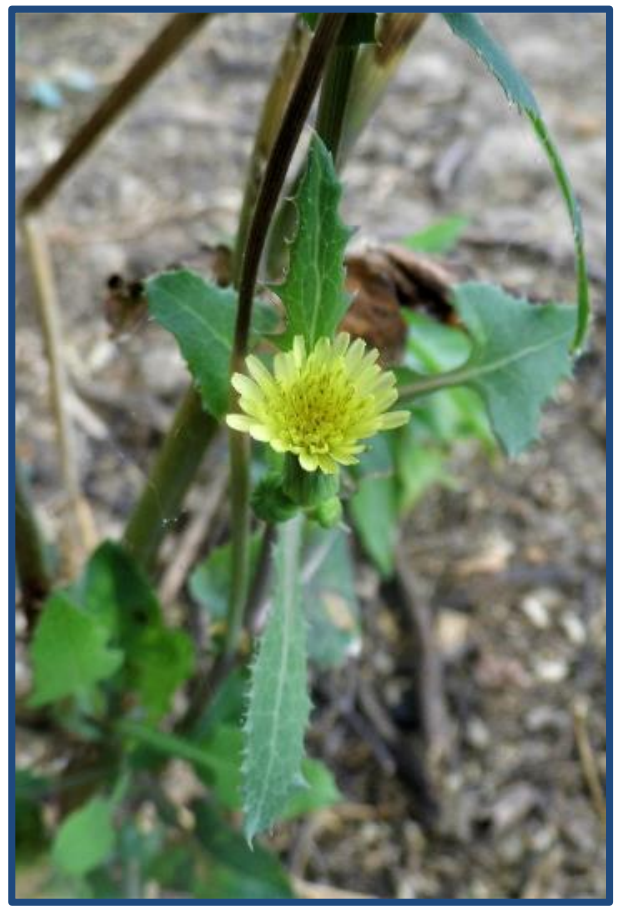

Figura 25: Spilanthes leiocarpa ASTERACEAE

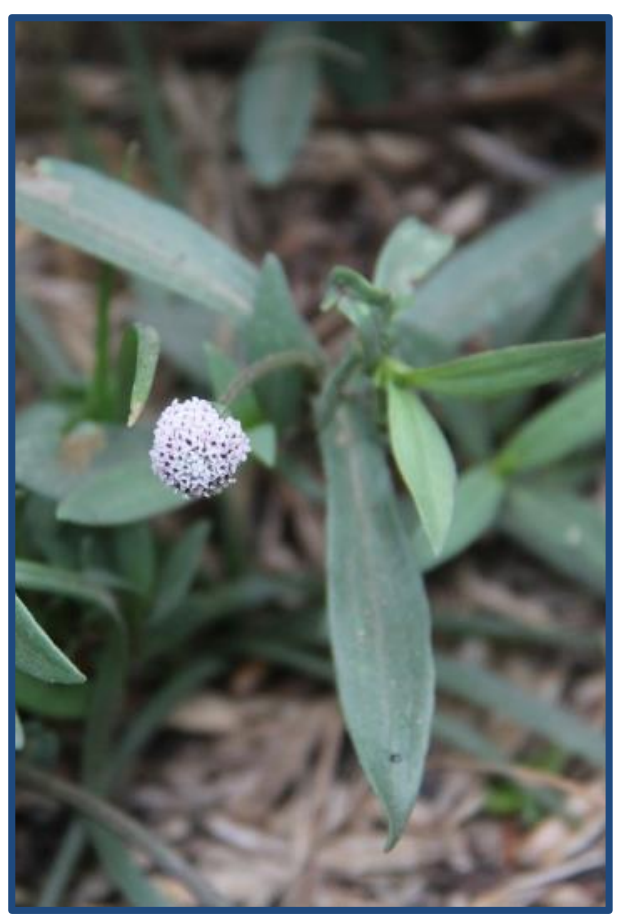

Figura 24: Sonchus oleraceus ASTERACEAE

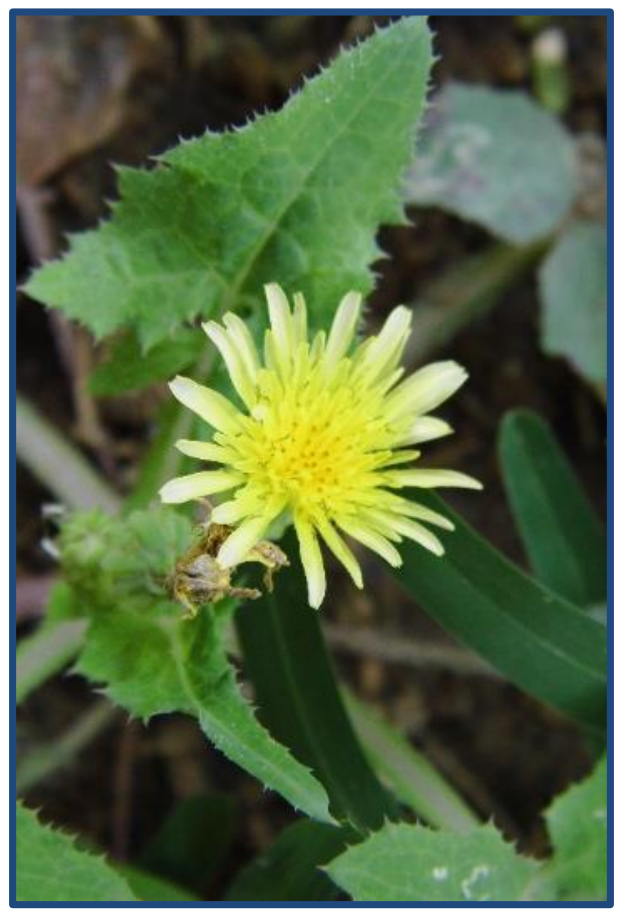

Figura 26: Spilanthes urens ASTERACEAE

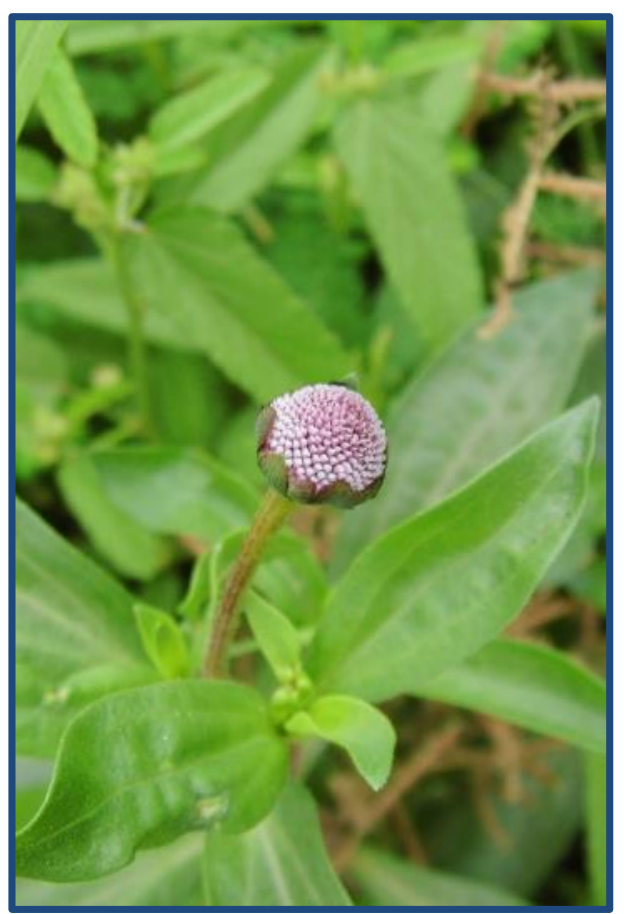


Figura 27: Tessaria integrifolia ASTERACEAE

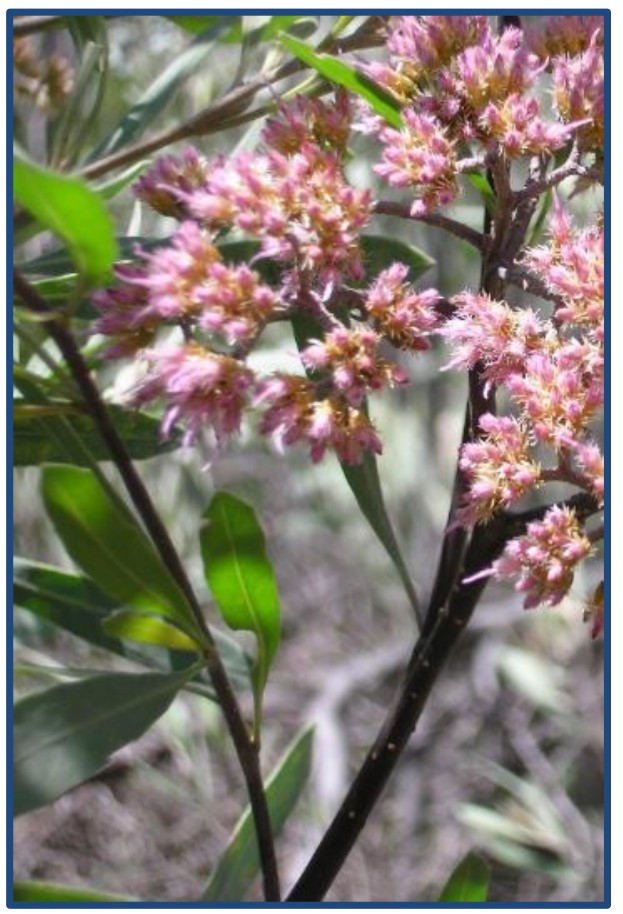

Figura 29: Proboscidea altheifolia MARTYNIACEAE

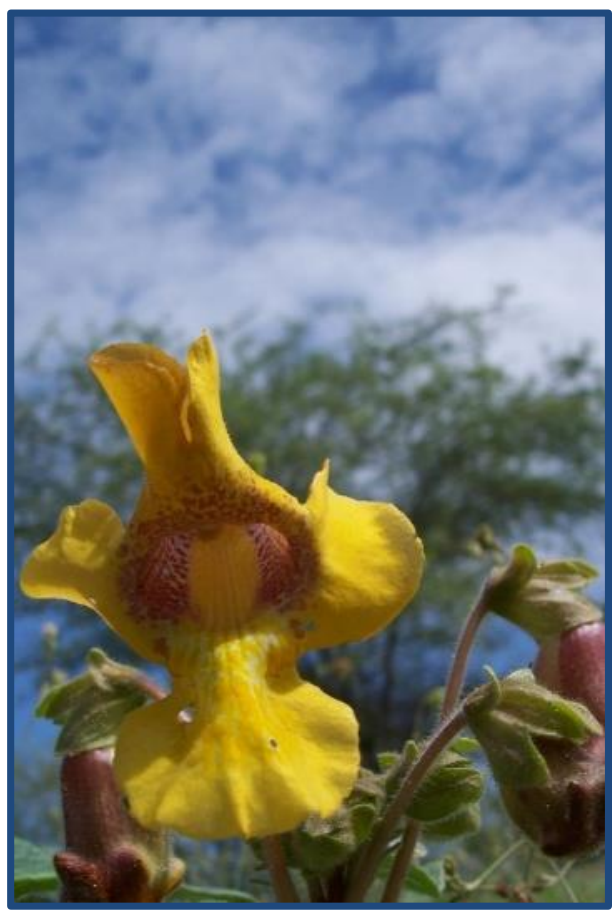

Figura 28: Momordica charantia CUCURBITACEAE

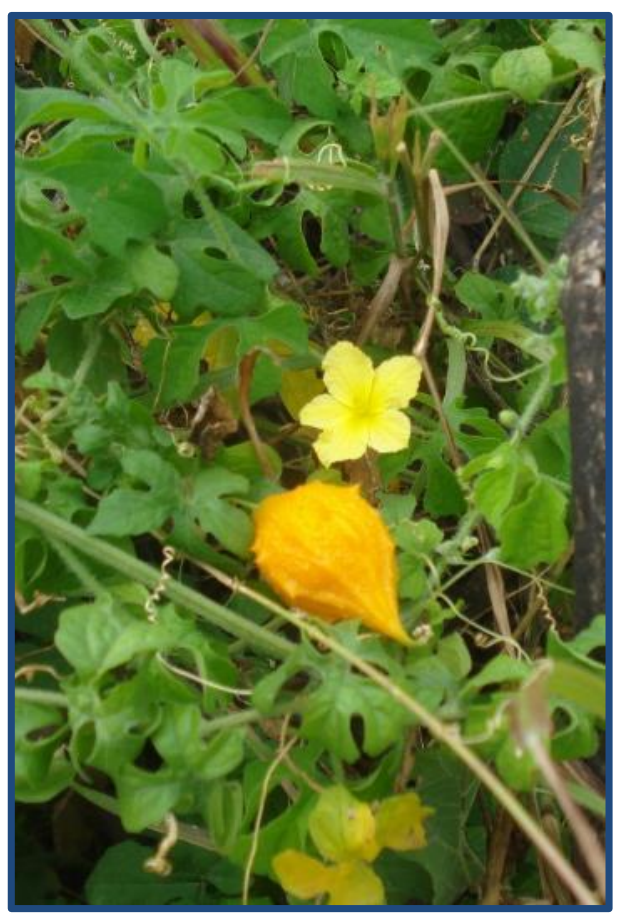

Figura 30: Cryptocarpus pyriformis NYCTAGINACEAE

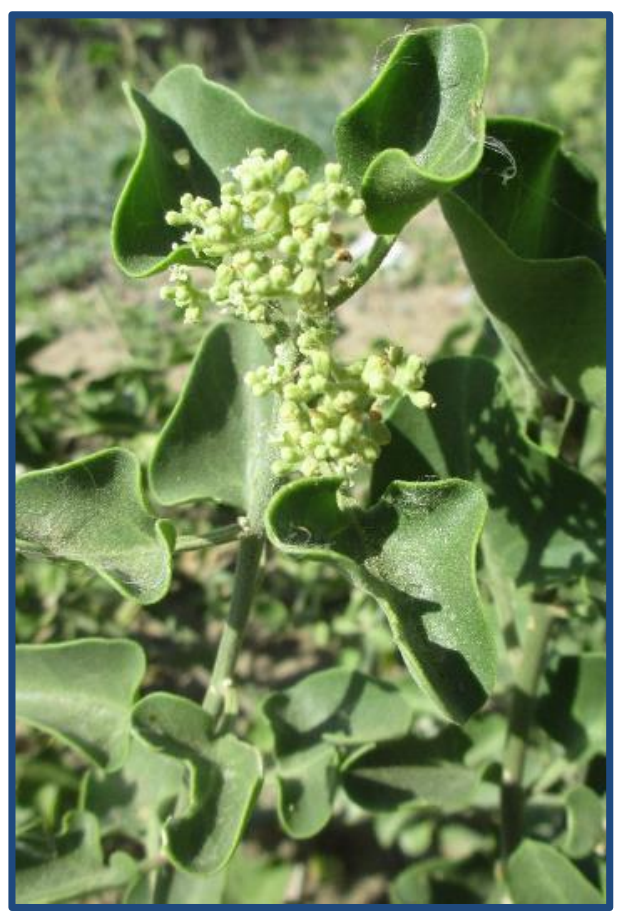

Ciencia Latina Revista Científica Multidisciplinar, Ciudad de México, México. ISSN 2707-2207 / ISSN 2707-2215 (en línea), marzo-abril, 2021, Volumen 5, Número 2. https://doi.org/10.37811/cl rcm.v5i2.337 p. 1437 
Figura 31: Acacia nilotica FABACEAE

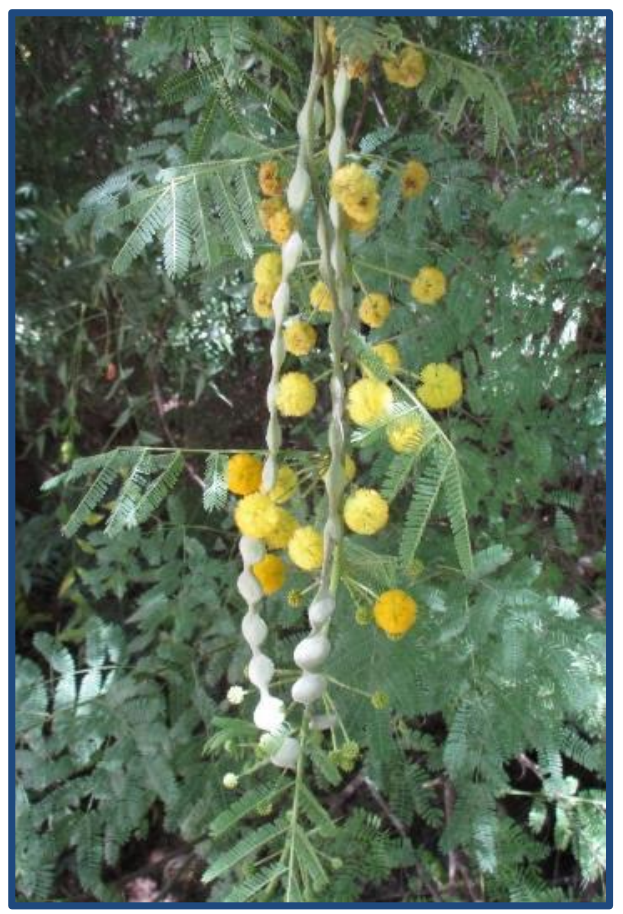

Figura 33: Desmanthus virgatus FABACEAE

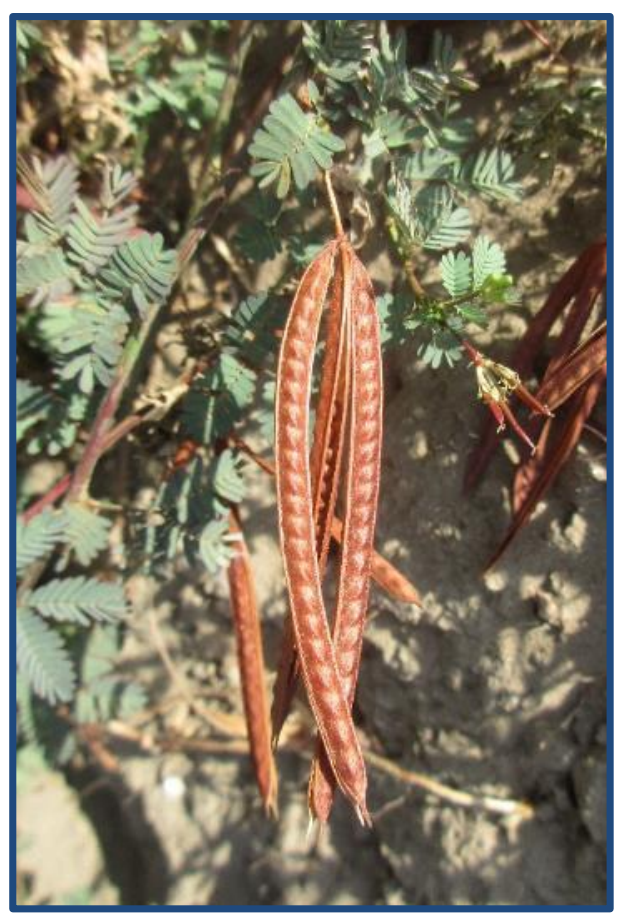

Figura 32: Vachellia macracantha FABACEAE

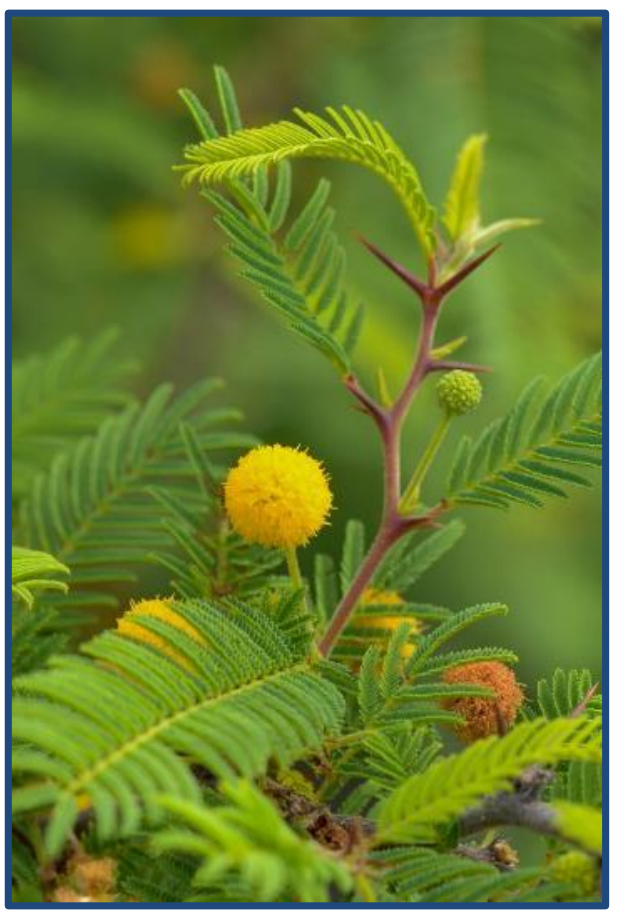

Figura 34: Macroptilium atropurpureum FABACEAE

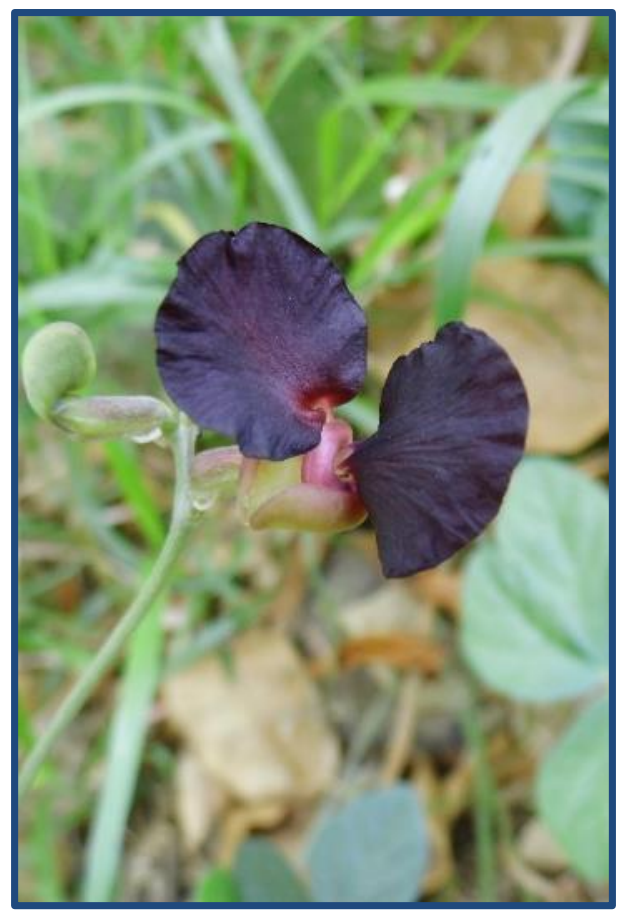

Ciencia Latina Revista Científica Multidisciplinar, Ciudad de México, México. ISSN 2707-2207 / ISSN 2707-2215 (en línea), marzo-abril, 2021, Volumen 5, Número 2. https://doi.org/10.37811/cl rcm.v5i2.337 p. 1438 
Figura 35: Parkinsonia aculeata FABACEAE

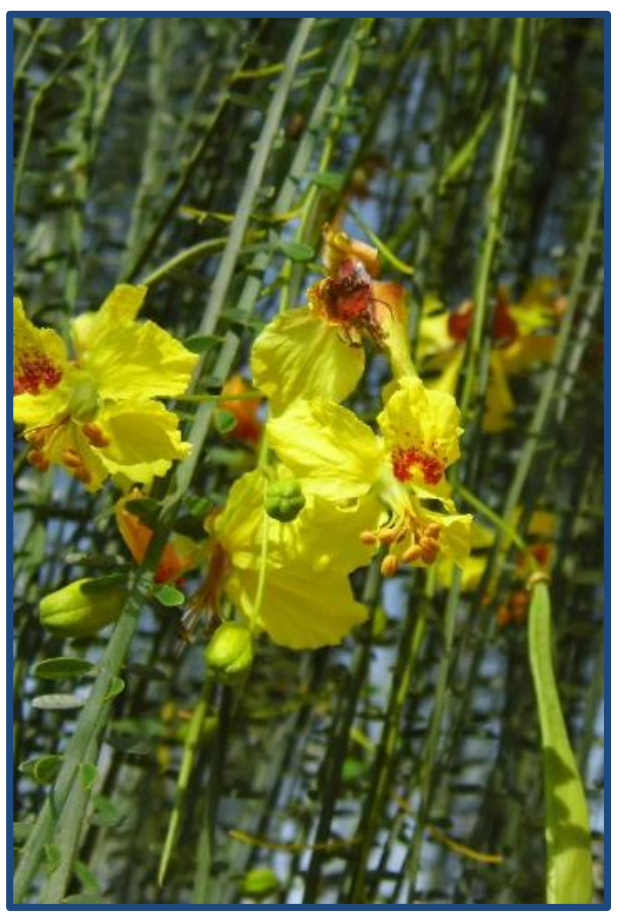

Figura 37: Solanum pimpinellifolium SOLANACEAE

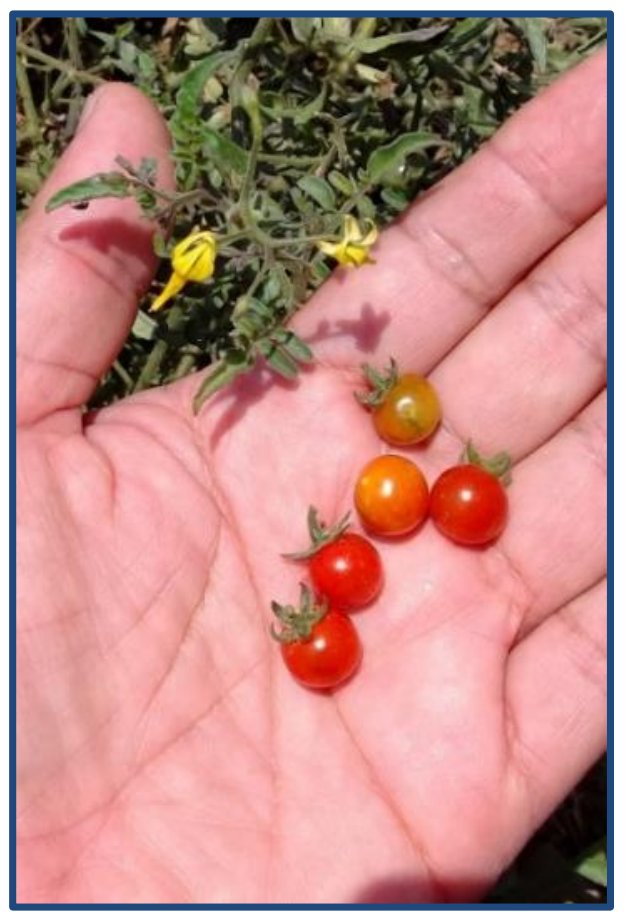

Figura 36: Prosopis pallida FABACEAE

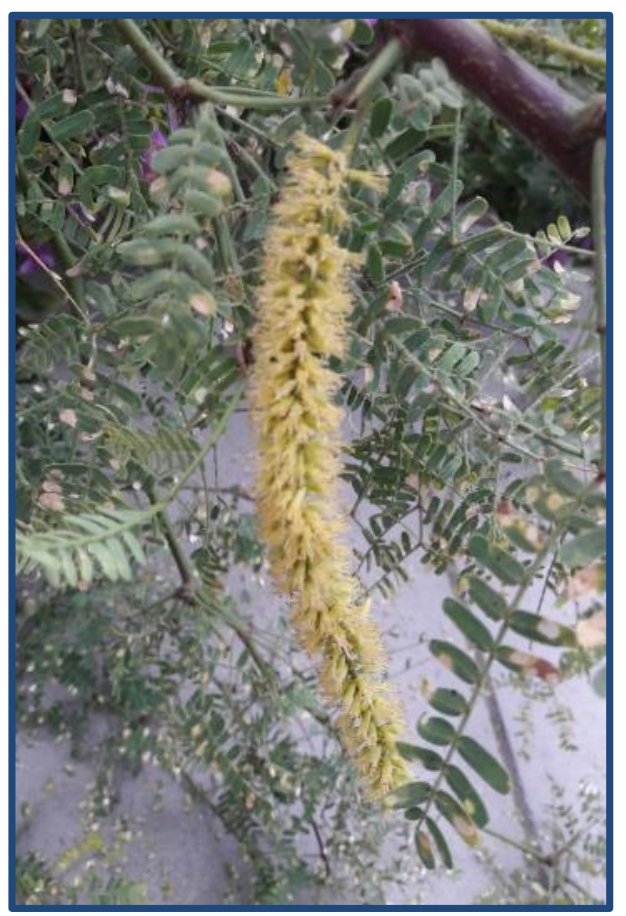

Figura 38: Grabowskia boerhaaviifolia SOLANACEAE

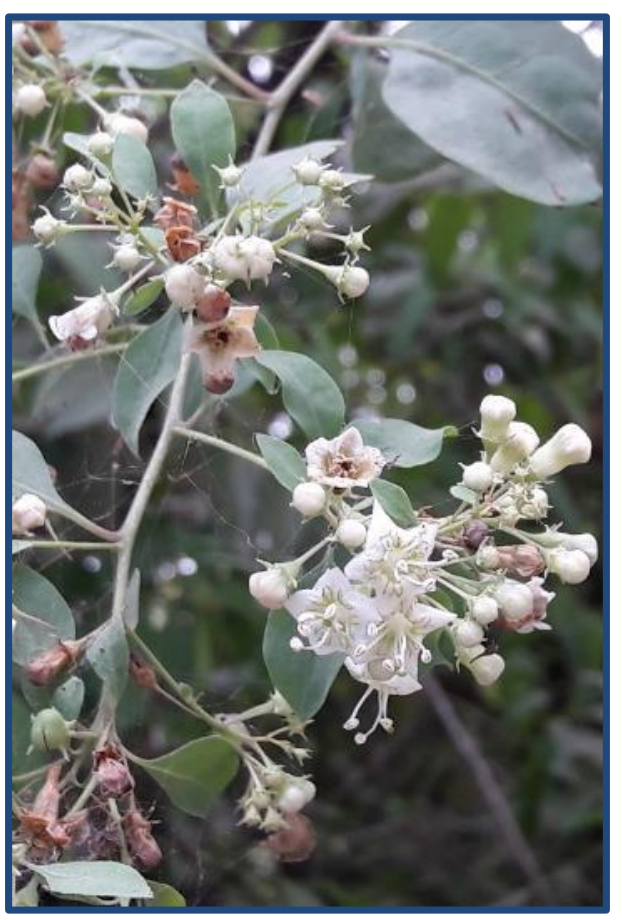

Ciencia Latina Revista Científica Multidisciplinar, Ciudad de México, México. ISSN 2707-2207 / ISSN 2707-2215 (en línea), marzo-abril, 2021, Volumen 5, Número 2. https://doi.org/10.37811/cl rcm.v5i2.337 p. 1439 
Figura 39: Bacopa monnieri PLANTAGINACEAE

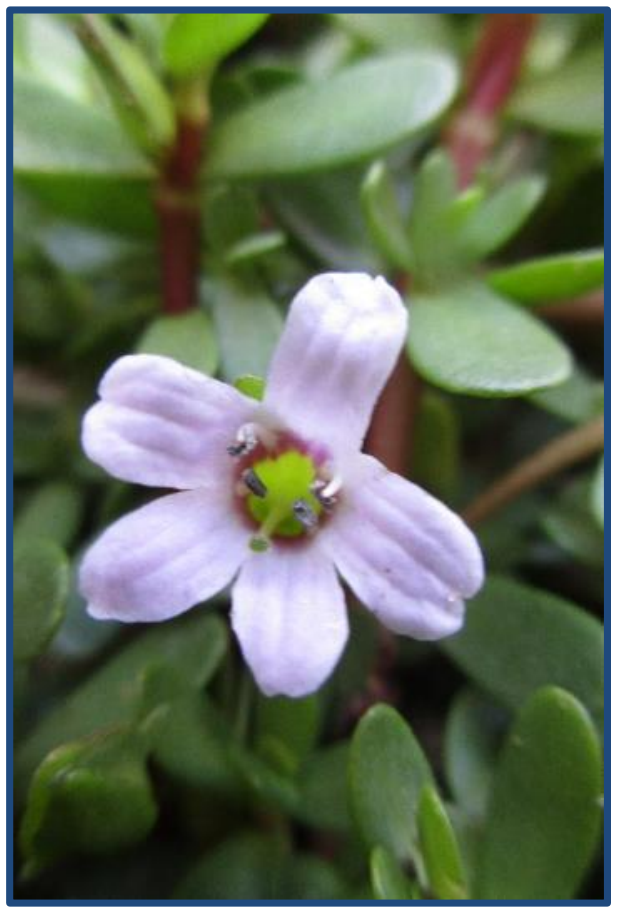

Figura 41: Tamarix aphylla TAMARICACEAE

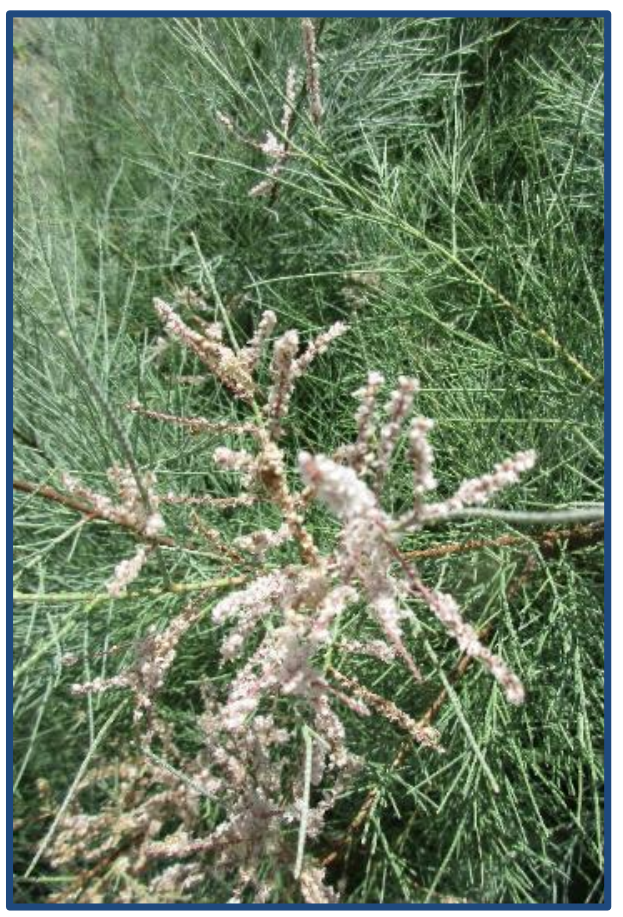

Figura 40: Portulaca oleracea PORTULACACEAE

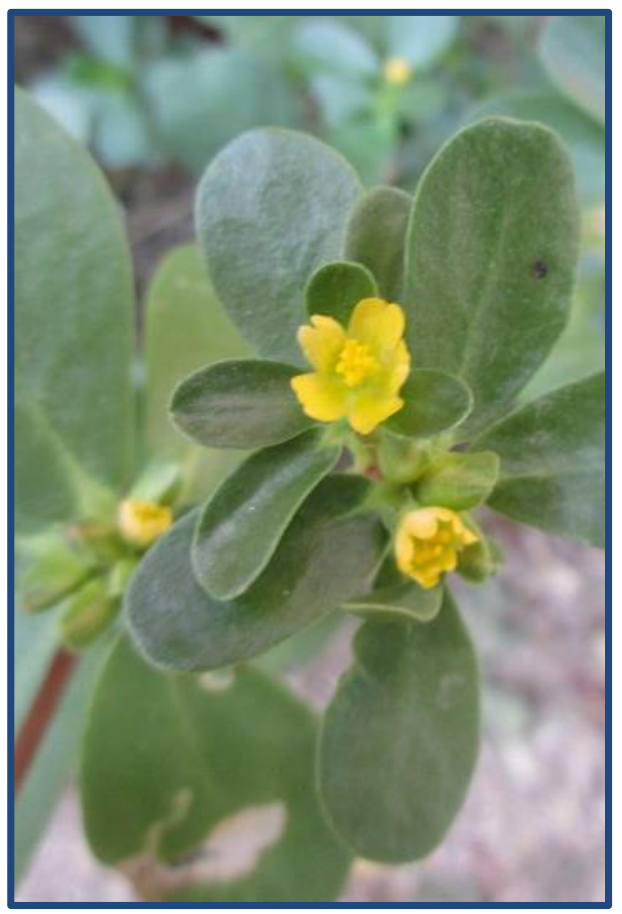

Figura 42: Tribulus terrestris ZYGOPHYLLACEAE

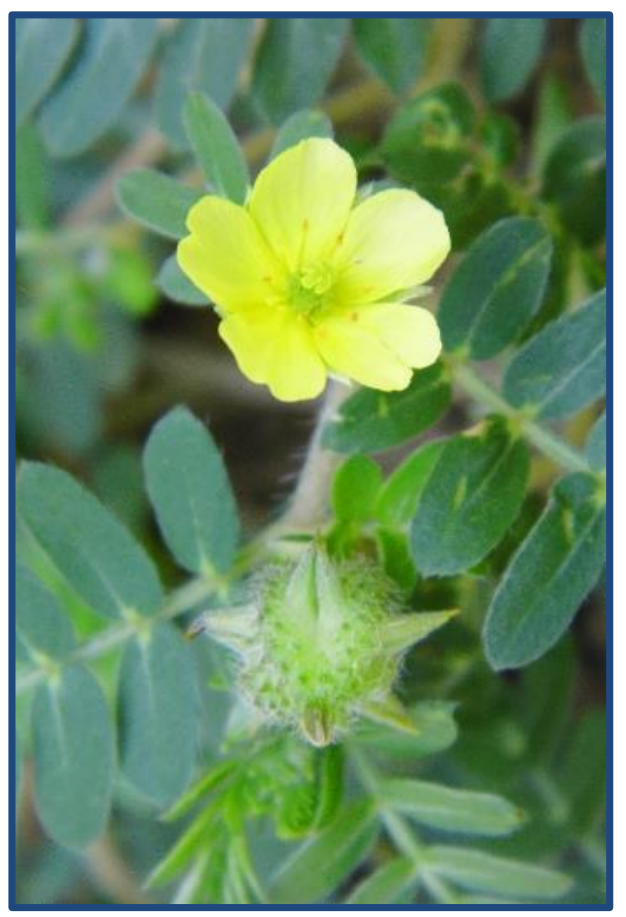

Ciencia Latina Revista Científica Multidisciplinar, Ciudad de México, México. ISSN 2707-2207 / ISSN 2707-2215 (en línea), marzo-abril, 2021, Volumen 5, Número 2. https://doi.org/10.37811/cl rem.v5i2.337 p. 1440 
Figura 43: Exodeconus maritimus SOLANACEAE

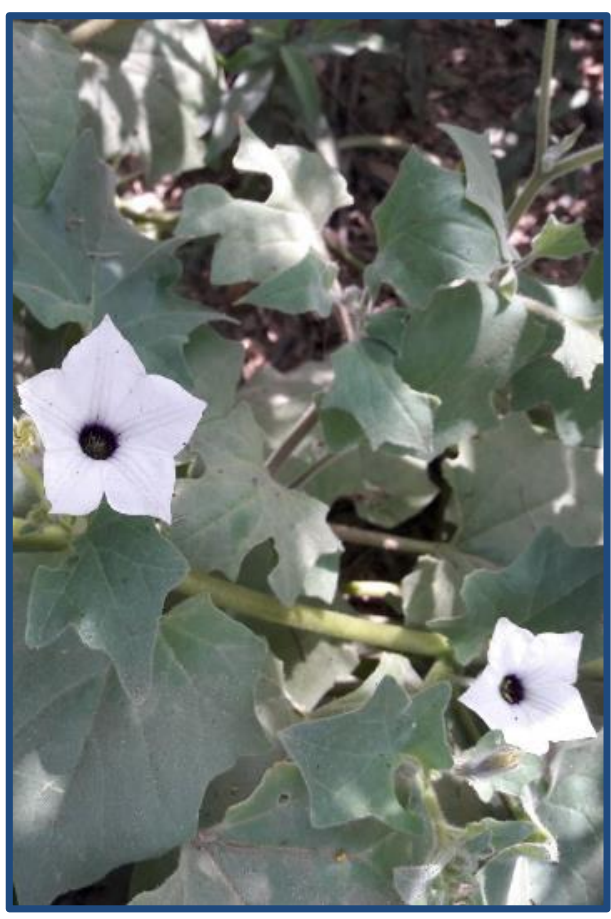

Figura 45: Cenchrus echinatus POACEAE

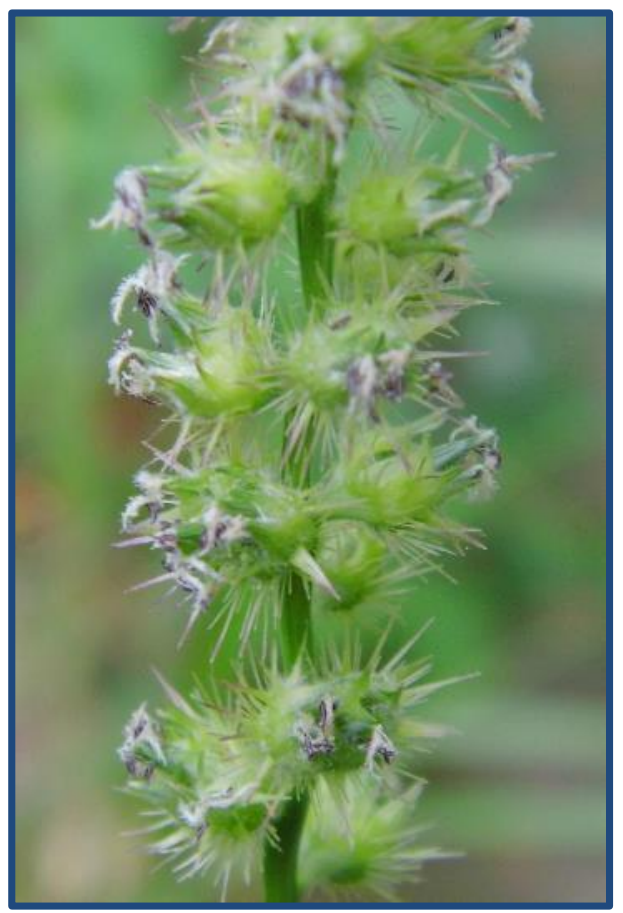

Figura 44: Typha angustifolia TYPHACEAE

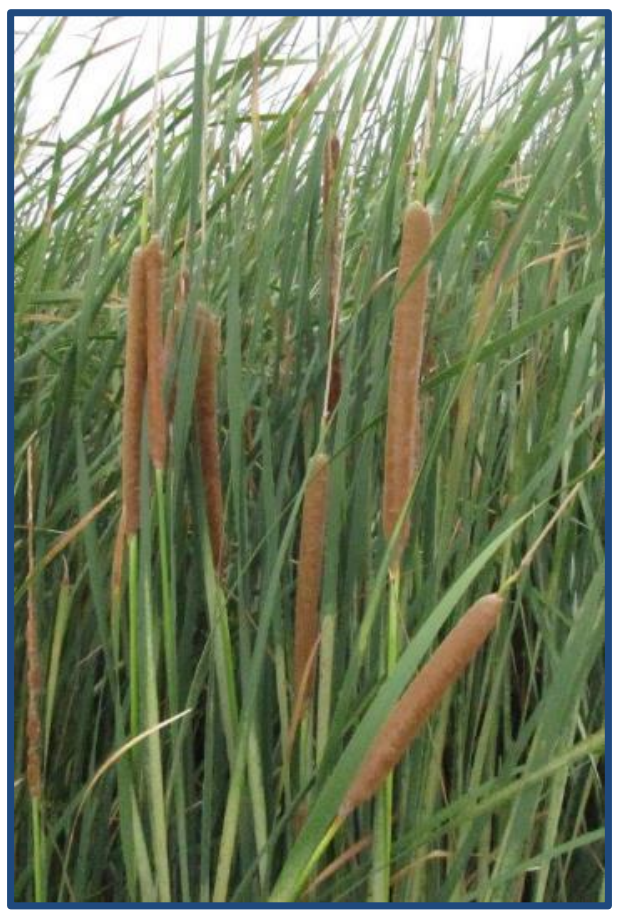

Figura 46: Cenchrus myosuroides POACEAE

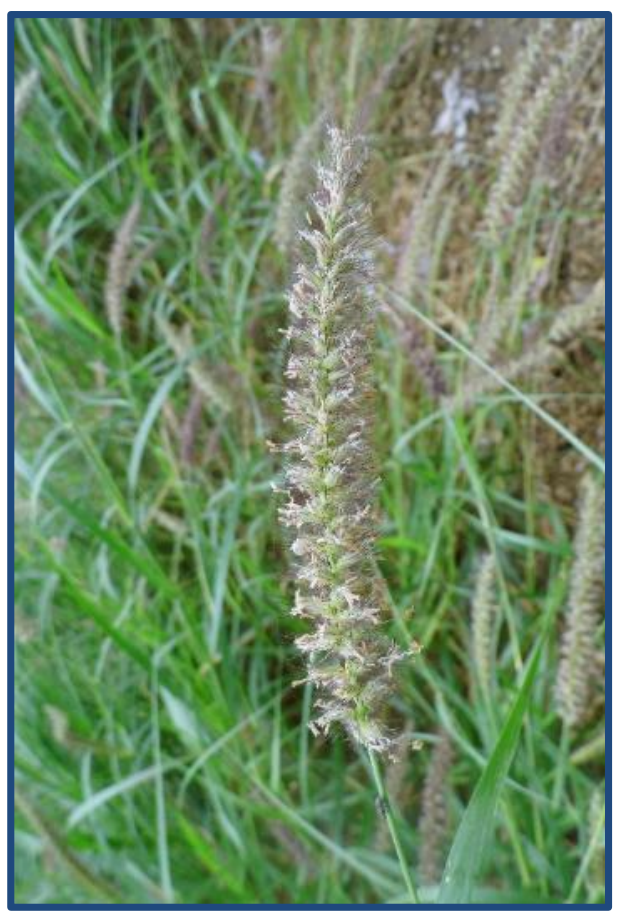

Ciencia Latina Revista Científica Multidisciplinar, Ciudad de México, México. ISSN 2707-2207 / ISSN 2707-2215 (en línea), marzo-abril, 2021, Volumen 5, Número 2. https://doi.org/10.37811/cl rcm.v5i2.337 p. 1441 
Figura 47: Cynodon dactylon POACEAE

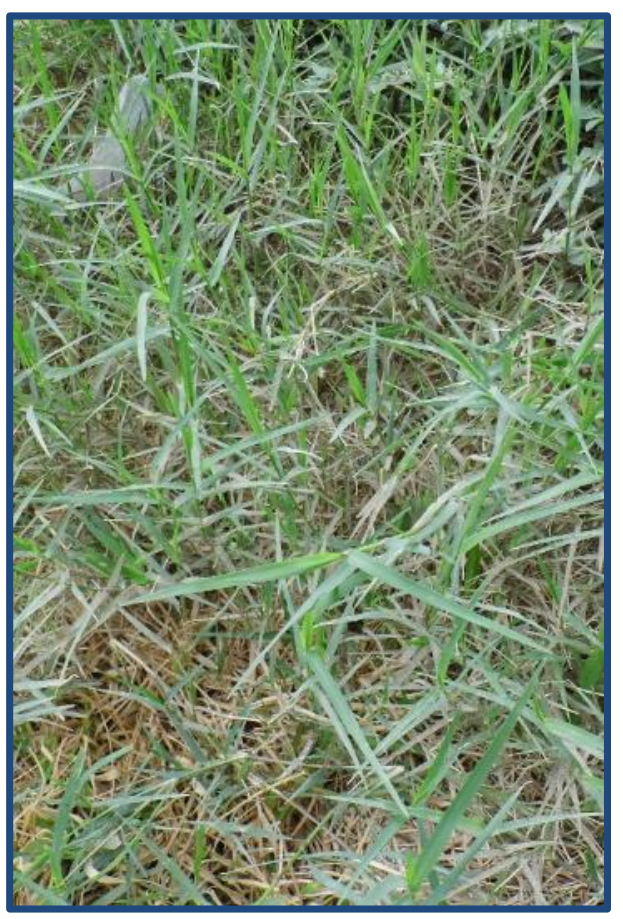

Figura 48: Dactyloctenium aegyptium POACEAE

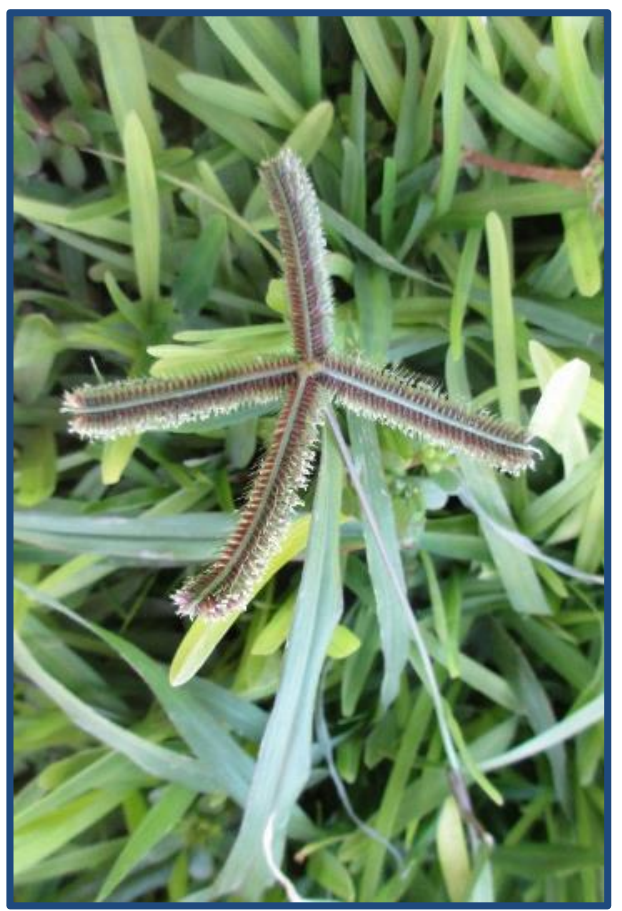

\section{CONCLUSIONES}

El Humedal de Castilla presenta 50 especies de magnoliófitas, contenidas en 42 géneros y 20 familias; 39 especies son nativas (78\%) y 11 especies introducidas (22\%). Esto nos indica la gran importancia de este ecosistema, que a pesar de tener un tamaño pequeño y de encontrarse en la zona de vida del desierto superárido - Premontano Tropical (ds-PT) de Perú, alberga un número mayor de especies vegetales que otros humedales costeros de mucho mayor tamaño ubicados en mejores zonas.

\section{LISTA DE REFERENCIAS}

APG IV (2016). The Linnean Society of London, Botanical Journal of the Linnean Society, 2016, 181, 1-20

Bridson D. \& L. Forman (1992). Herbarium Handbook. Royal Botanical Gardens, Kew.

Butler S., ed. (2010). Macquarie Concise Dictionary (5th ed.). Sydney Australia: Macquarie Dictionary Publishers Ltd. ISBN 978-1-876429-85-0.

CDB - Convenio sobre la Diversidad Biológica (2020, febrero 02). Especies Exóticas Invasoras. Secretaría del Convenio sobre la Diversidad Biológica. Montreal, Quebec Canada. https://www.cbd.int/undb/media/factsheets/undb-factsheet-ias-es.pdf 
Charcape Ravelo, Manuel \& Moutarde, Fanny (2005). Diversidad Florística y Conservación del Santuario Regional de Piura Manglares San Pedro de Vice-Sechura. Revista Peruana de Biología 12 (2), 327-34. https://doi.org/10.15381/rpb.v12i2.2406 .

Cowardin, Lewis M.; Carter, Virginia; Golet, Francis C. \& LaRoe, Edward T. (2021, febrero 10). Classification of wetlands and deepwater habitats of the United States. Wetlands and Deepwater Habitats. U.S. Department of the Interior, Fish and Wildlife Service, Washington, D.C. Jamestown, ND: Northern Prairie Wildlife Research Center Online. https://www.fws.gov/wetlands/documents/classwet/wetlands.htm

Kusler J.A., W.J. Mitsch \& J.S. Larson (1994). Humedales. Investigación y Ciencia 210: 6-13.

León, Blanca (1993). Catálogo anotado de las fanerógamas acuáticas del Perú. Las Plantas Vasculares en las Aguas Continentales del Perú. Travaux de l'Institut Francais d'Etudes Andines. (eds F. Kahn, B. León, \& K. Young), IFEA (Institut Francais d'Etudes Andines), Lima-Perú.

León, Blanca; Cano, Asunción \& Young, Kenneth R. (1995). La flora vascular de los Pantanos de Villa, Lima, Perú. Adiciones y guía para las especies comunes. Publicaciones del Museo de Historia Natural-UNMSM (B) 38: 1-39.

Mitsch, William J. \& James G. Gosselink (2007). Wetlands (4th ed.). New York: John Wiley \& Sons. ISBN 978-0-471-69967-5.

Monzón Licera, Katia L. \& Peláez Peláez, Freddy (2015). Flora vascular de los humedales Tres Palos, Ascope, Perú, 2013. REBIOL 2015; 35(1): 108-116.

Mostacero León, José; Mejía Coico, Freddy \& Gamarra Torres, Oscar (2009). Fanerógamas del Perú: taxonomía, utilidad y ecogeografía. Aupiciada por el Consejo Nacional de Ciencia y Tecnología. Edit. Normas Legales. Trujillo - Perú. ISBN: 9789972-50-099-2

ProNaturaleza (2010). HUMEDALES DE LA COSTA PERUANA. documento base para la elaboración de una estrategia de conservación. G \& G Impresores SAC. Lima. Perú. ISBN: 978-612-45697-1-5

Ramsar (1971). Manual de la Convención de Ramsar. Guía a la Convención sobre los Humedales. Gland (Suiza): Secretaría de la Convención de Ramsar - Irán.

Ramsar (2021, febrero 05). Perspectiva Mundial sobre los humedales. Estado de los humedales del mundo y servicios que prestan a las personas. Secretaría de la 
Convención

de

Ramsar.

https://www.ramsar.org/sites/default/files/documents/library/gwo_s.pdf

RBG - Royal Botanic Gardens (2021, febrero 01). The State of the World's Plants. RBG

Kew. https://stateoftheworldsplants.org/2016/report/sotwp_2016.pdf

SERFOR - Servicio Forestal y de Fauna Silvestre (2021, febrero 10). Humedales ecosistemas para la vida. Ministerio de Agricultura y Riego. Lima Perú. https://www.minam.gob.pe/wp-content/uploads/2015/02/DMH-2015-

presentaci\%C3\%B3n-institucional-SERFOR_Fmu\%C3\%B1oz.pdf

SOTWP - The State of The World's Plants (2021, febrero 08). Naming and counting the world's plant families. 4 Describing the world's plants. https://stateoftheworldsplants.org/2017/report/SOTWP_2017_1_naming_and_counti ng_the_world_s_plant_families.pdf

Tosi, Joseph A. (1960). Zonas de Vida Natural en el Perú. Memoria explicativa sobre el mapa ecológico del Perú. Instituto Interamericano de Ciencias Agrícolas de la OEA Zona Andina. Boletín técnico n 5. 